University of Louisville

ThinkIR: The University of Louisville's Institutional Repository

$5-2014$

\title{
Effect of metallic restoration artifacts on maxillofacial cone beam computed tomography images.
}

Mitali Binani 1987-

University of Louisville

Follow this and additional works at: https://ir.library.louisville.edu/etd

Part of the Oral Biology and Oral Pathology Commons

\section{Recommended Citation}

Binani, Mitali 1987-, "Effect of metallic restoration artifacts on maxillofacial cone beam computed tomography images." (2014). Electronic Theses and Dissertations. Paper 110.

https://doi.org/10.18297/etd/110

This Master's Thesis is brought to you for free and open access by ThinkIR: The University of Louisville's Institutional Repository. It has been accepted for inclusion in Electronic Theses and Dissertations by an authorized administrator of ThinkIR: The University of Louisville's Institutional Repository. This title appears here courtesy of the author, who has retained all other copyrights. For more information, please contact thinkir@louisville.edu. 


\title{
EFFECT OF METALLIC RESTORATION ARTIFACTS ON MAXILLOFACIAL CONE BEAM COMPUTED TOMOGRAPHY IMAGES
}

\author{
By \\ Mitali Binani \\ M.S, University of Louisville, 2014
}

\begin{abstract}
A Thesis
Submitted to the Faculty of the School of Dentistry of the University of Louisville In Partial Fulfillment of the Requirements

For the Degree of
\end{abstract}

Master of Science

Oral Biology

Louisville, Kentucky

May, 2014 



\title{
EFFECT OF METALLIC RESTORATION ARTIFACTS ON MAXILLOFACIAL CONE BEAM COMPUTED TOMOGRAPHY IMAGES.
}

\author{
By \\ Mitali Binani \\ M.S, University of Louisville, 2014
}

A Thesis Approved on

April 17, 2014

By the following Thesis Committee:

Dr. Allan G. Farman, Thesis Co-Director

Dr. William C. Scarfe, Thesis Co-Director

Dr. Bryan T. Harris, Committe Member 


\section{DEDICATION}

This thesis is dedicated to my family and friends for their love and support. Without their encouragement, this thesis would not have been possible. 


\section{ACKNOWLEDGEMENTS}

Dr. Allan G Farman, Thesis Co-Director, for providing me the opportunity to work under his guidance, and for contributing to this research project with his immense expertise in the specialty of Oral and Maxillofacial Radiology.

Dr. William C Scarfe, Thesis Co-Director, for his constant support and enthusiasm in all phases of the research. It was his relentless mentorship that made this project a successful endeavor.

Dr. Bryan T Harris, Thesis Committee Member, for his help to refine this project with his knowledge. 


\title{
ABSTRACT
}

\section{EFFECT OF METALLIC RESTORATION ARTIFACTS ON MAXILLOFACIAL CONE BEAM COMPUTED TOMOGRAPHY IMAGES.}

\author{
Mitali Binani
}

April 17, 2014

Artifacts due to high-density objects (HDO) such as metallic dental restorations on maxillofacial cone beam computed tomographic (CBCT) images can render certain areas unsuitable for diagnosis. It was hypothesized that image quality due to HDO artifacts was affected by CBCT acquisition parameters and the number and configuration of HDO. Simulated complete maxillary and mandibular dental arches were constructed using dental stone and extracted teeth. Conservative coronal dental amalgam (MOD) restorations on premolar and molar teeth were used as HDOs. Gray values $(\mathrm{GV})$ measured on uniform dental stone test cylinders at specific levels from the occlusal plane at three tooth locations was used as an index of artifact effect on image quality. Scans with various HDO configurations were taken at several acquisition parameters for three CBCT systems: Accuitomo 170 (J. Morita MFG. Corp, Kyoto, Japan), iCAT Next Generation (Imaging Sciences International Inc., Hatfield, PA, USA) and Carestream 9000 3D (Carestream/KODAK, Atlanta, GA). For all systems, HDOs significantly affected images throughout the field of view, with machine specific beam hardening or scatter artifacts. Worst beam hardening affected areas were within $0 \mathrm{~mm}-4 \mathrm{~mm}$ of the occlusal plane of the ipsilateral test cylinder. The Accuitomo 170 was unaffected by 
acquisition parameters. Caution must be exercised when assessing CBCT images for coronal dental caries and other pathologies in the presence of HDOs to prevent errors in diagnosis due to beam hardening or scatter artifacts. 
TABLE OF CONTENTS

$P A G E$

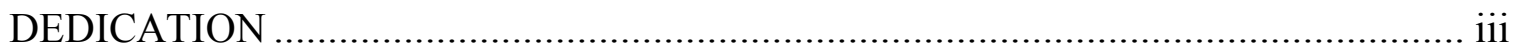

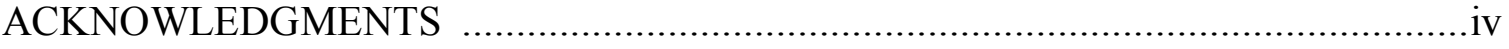

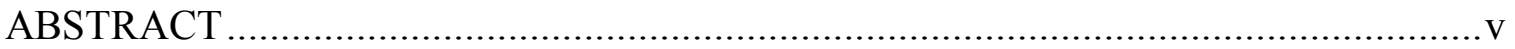

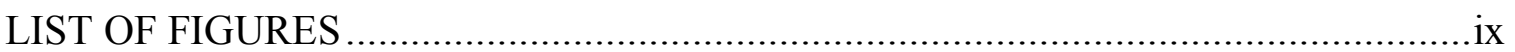

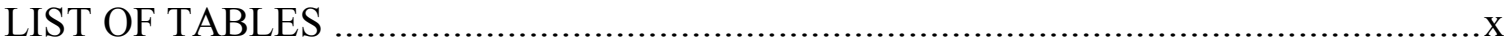

INTRODUCTION AND LITERATURE REVIEW ................................................

HYPOTHESIS …

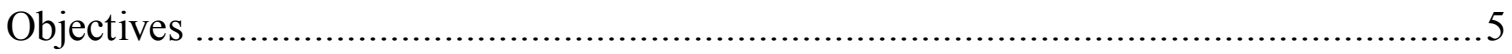

Null Hypothesis .............................................................................................

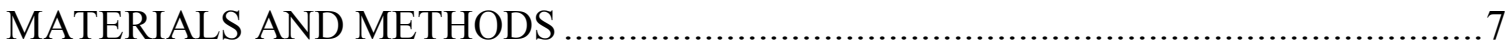

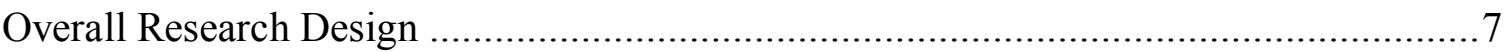

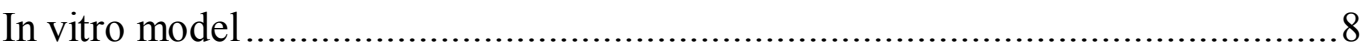

High Density Object (HDO) Configuration.................................................... 10

Cone Beam Computed Tomographic Imaging ……………........................... 10

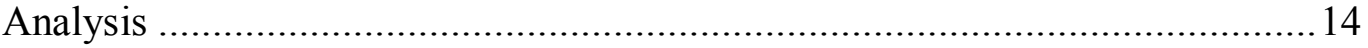

CBCT Image Evaluation ...................................................... 14

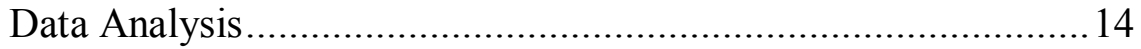

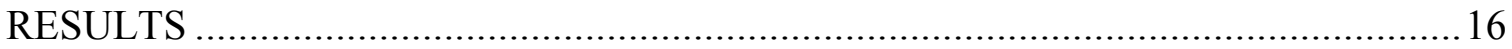

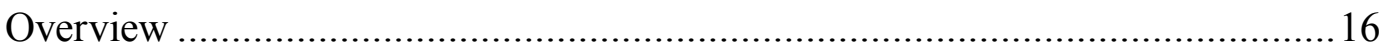

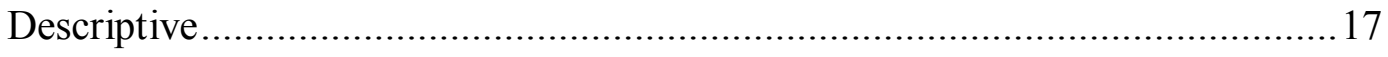

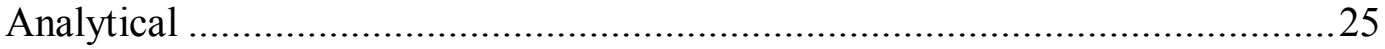

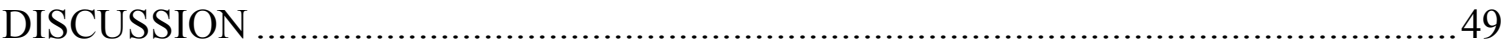

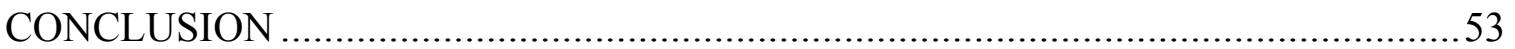


REFERENCES.

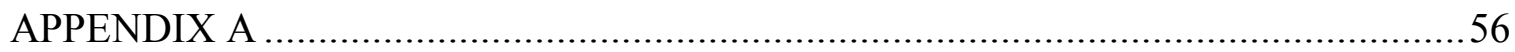

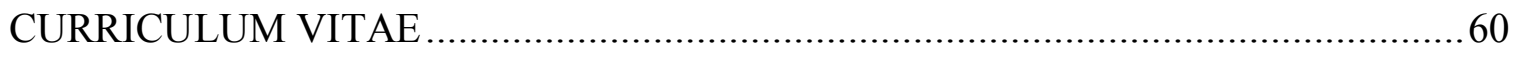




\section{LIST OF FIGURES}

FIGURE

$P A G E$

1. CS 9000 interval plot showing $\Delta \mathrm{GV} \%$ for each test cylinder location according to each independent variable (Depth, $\mathrm{kV}$ and resolution)

2. CS 9000 interval plot of $\Delta \mathrm{GV} \%$ (y-axis) according to position of the HDO

relative to the test cylinder (adjacent vs. non-adjacent) for the CS 9000D

3. iCAT NG interval plot showing $\Delta \mathrm{GV} \%$ for each test cylinder location according to each independent variable (Depth, $\mathrm{kV}$ and resolution).

4. iCAT NG interval plot showing $\Delta \mathrm{GV} \%$ for each test cylinder location according to each independent variable (Depth, $\mathrm{kV}$ and resolution) according to position of the HDO relative to the test cylinder (adjacent vs. nonadjacent).

5. 3D Accuitomo 170 interval plot showing $\Delta \mathrm{GV} \%$ for each test cylinder location according to each independent variable (Depth, $\mathrm{kV}$ and resolution). .23

6. 3D Accuitomo 170 interval plot showing $\Delta \mathrm{GV} \%$ for each test cylinder location according to each independent variable (Depth, $\mathrm{kV}$ and resolution) according to position of the HDO relative to the test cylinder (adjacent vs. nonadjacent). 


\section{LIST OF TABLES}

1. Summary of Studies on the Diagnostic Accuracy of CBCT in the Detection of Dental Caries

2. Comparison of gray values of dentin in the control model (without HDO) and patient radiographs (without HDO) on iCAT Next Generation CBCT Unit (120 kV / 5 mA)

3. Gray values of dentin on control model on Accuitomo 170 and

4. Location of High Density Objects

5. Exposure parameters for the Carestream 9000 3D CBCT System

6. Exposure parameters for the iCAT Next Generation CBCT System 12

7. Exposure parameters for the Acciutomo 170 CBCT System.

8. Choosing the Appropriate Multiple Comparison Test - Normality (Anderson-Darling test) And Equal Variance Tests for independent variables for each CBCT unit

9. CS 90003D GLM

10. Post Hoc pairwise comparison (Tukey) for $\Delta \mathrm{GV} \%$ for various configurations of HDO Artifact

11. Post Hoc pairwise comparison (Tukey) for $\Delta \mathrm{GV} \%$ at various level of cylinder .. 30

12. Post Hoc pairwise comparison (Tukey) for $\Delta \mathrm{GV} \%$ at various arch locations .......31

13. Post Hoc pairwise comparison (Tukey) for $\Delta \mathrm{GV} \%$ at two $\mathrm{kV}$ levels.

14. Post Hoc pairwise comparison (Tukey) for $\Delta \mathrm{GV} \%$ at high and low resolutions ...31

15. Post Hoc pairwise comparison (Tukey) for $\Delta \mathrm{GV} \%$ according to the side where HDO is positioned. 
16. Post Hoc pairwise comparison (Tukey) for $\Delta \mathrm{GV} \%$ at position of $\mathrm{HDMO}$ relative to test cylinder

17. Analysis of Variance for iCAT Next Generation ................................................ 33

18. Post Hoc pairwise comparison (Tukey) for $\Delta \mathrm{GV} \%$ for various configurations of

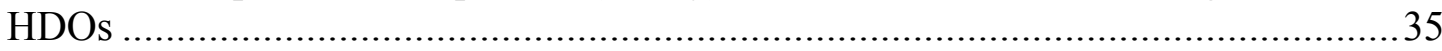

19. Post Hoc pairwise comparison (Tukey) for $\Delta \mathrm{GV} \%$ at various level of cylinder ..38 20. Post Hoc pairwise comparison (Tukey) for $\Delta \mathrm{GV} \%$ at various arch locations .......39

21. Post Hoc pairwise comparison (Tukey) for $\Delta \mathrm{GV} \%$ at two resolutions. .................39

22. Post Hoc pairwise comparison (Tukey) for $\Delta \mathrm{GV} \%$ at two arches .........................39

23. Post Hoc pairwise comparison (Tukey) for $\Delta \mathrm{GV} \%$ according to side of $\mathrm{HDO} \ldots . .40$

24. Post Hoc pairwise comparison (Tukey) for $\Delta \mathrm{GV} \%$ according to proximity of

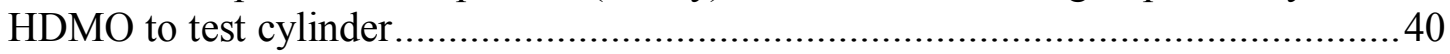

25. 3D Accuitomo 170 Analysis of Variance ...........................................................4

26. Post Hoc pairwise comparison (Tukey) for $\Delta \mathrm{GV} \%$ for various configurations of

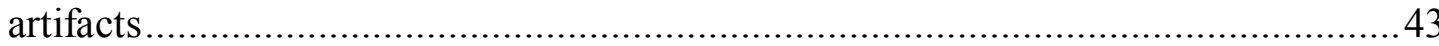

27. Post Hoc pairwise comparison (Tukey) for $\Delta \mathrm{GV} \%$ at various levels of cylinder. 46 28. Post Hoc pairwise comparison (Tukey) for $\Delta \mathrm{GV} \%$ at various arch locations .......47

29. Post Hoc pairwise comparison (Tukey) for $\Delta \mathrm{GV} \%$ at two arches ........................47

30. Post Hoc pairwise comparison (Tukey) for $\Delta \mathrm{GV} \%$ according to side of HDO in

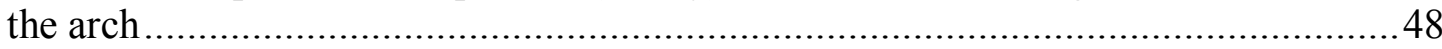

31. Post Hoc pairwise comparison (Tukey) for $\Delta \mathrm{GV} \%$ according to proximity of HDO to test cylinder .48 


\section{CHAPTER I}

\section{INTRODUCTION AND LITERATURE REVIEW}

Maxillofacial cone beam computed tomography (CBCT) has helped improve diagnosis, treatment planning and follow up with much more accurate and precise imaging. However, there are still some inherent artifacts produced that limit the use of this technology for specific tasks. These introduce errors that reduce image quality and should be considered with clinical use.

The most common patient related artifacts produced in the field of view (FOV) of CBCT are as a result of the presence of high-density objects (HDO) such as amalgam, composite resin, implants, etc. Attenuation and interaction of x-rays with HDO produce scatter, beam hardening, and photon starvation and produce images with dark and light streak artifacts. ${ }^{(1)}$ These artifacts are more intense in the mesio-distal region of the $\mathrm{HDO}^{(2,}$

3) and may be severe enough so as to render the scans unsuitable for interpretation. In a retrospective, observational study, Ritter, et al. ${ }^{(4)}$ concluded that restorations negatively impact the image quality of CBCT images. They specifically used the Galileos CBCT system (Sirona Dental Systems, Bensheim, Germany). Since much of the population have dental amalgam restorations, it is important to quantitatively assess the effect of the presence of dental amalgam with the pattern and intensity of artifacts produced and ultimately correlate this with the diagnostic yield of CBCT specifically for the assessment of coronal conditions such as the detection of the presence of dental caries. 
There is significant controversy regarding the assertion that $\mathrm{CBCT}$ is potentially a better diagnostic imaging tool for coronal dental caries than conventional radiography (Table 1).

Table 1. Summary of Studies on the Diagnostic Accuracy of CBCT in the Detection of Dental Caries

\begin{tabular}{|c|c|c|c|c|c|c|}
\hline \multirow{3}{*}{$\begin{array}{l}\text { Author } \\
\text { reference }\end{array}$} & \multirow{3}{*}{ Year } & \multicolumn{2}{|l|}{ Caries } & \multicolumn{2}{|c|}{ Modality } & \multirow{3}{*}{ Results } \\
\hline & & $\begin{array}{c}\text { Type } \\
\text { of } \\
\text { teeth }\end{array}$ & $\begin{array}{l}\text { Surf } \\
\text { ace }\end{array}$ & \multirow[t]{2}{*}{ CBCT } & \multirow[t]{2}{*}{ Intraoral } & \\
\hline & & $D / P$ & $\begin{array}{l}\text { Occ / } \\
\text { Prox }\end{array}$ & & & \\
\hline (5) & 2006 & $\mathrm{P}$ & Prox & $\begin{array}{l}\text { Limited cone beam } \\
\text { computed } \\
\text { tomography } \\
\text { (LCBCT) }\end{array}$ & $\begin{array}{l}\text { Image plate } \\
\text { system and } \\
\text { F-speed film }\end{array}$ & $\begin{array}{l}\text { LCBT fared better } \\
\text { than intra oral } \\
\text { radiography in } \\
\text { detecting proximal } \\
\text { caries. }\end{array}$ \\
\hline (6) & 2007 & $\mathrm{P}$ & Prox & $\begin{array}{l}\text { 3D Accuitomo (J. } \\
\text { Morita Mfg. Corp., } \\
\text { Kyoto, Japan) }\end{array}$ & $\begin{array}{l}\text { Insight films } \\
\text { (Eastman } \\
\text { Kodak, } \\
\text { Rochester, } \\
\text { NY) }\end{array}$ & $\begin{array}{l}\text { No difference } \\
\text { between accuracy in } \\
\text { detecting proximal } \\
\text { carious lesion }\end{array}$ \\
\hline (7) & 2008 & $\mathrm{P}$ & Both & $\begin{array}{l}\text { NewTom 3G and } \\
\text { 3DX Accuitomo }\end{array}$ & $\begin{array}{l}\text { Digora-fmx } \\
\text { and Kodak } \\
\text { Insight films }\end{array}$ & $\begin{array}{l}\text { NewTom } 3 \mathrm{G} \text { has } \\
\text { lower diagnostic } \\
\text { accuracy than others. }\end{array}$ \\
\hline (8) & 2008 & $\begin{array}{l}\text { CBCT } \\
\text { when } n\end{array}$ & $\begin{array}{l}\text { ystems } \\
\text { metal }\end{array}$ & $\begin{array}{l}\text { re better than intraor } \\
\text { elements present in }\end{array}$ & $\begin{array}{l}\text { systems at det } \\
\text { he oral cavity. }\end{array}$ & cting carious lesions \\
\hline (9) & 2009 & $\mathrm{P}$ & Both & 3DX Accuitomo & $\begin{array}{l}\text { Gendex } 1000 \\
\text { X-ray unit }\end{array}$ & $\begin{array}{l}\text { 3DX high resolution } \\
\text { CBCT fare better in } \\
\text { detecting proximal } \\
\text { caries but not } \\
\text { occlusal caries when } \\
\text { compared with CCD } \\
\text { images. }\end{array}$ \\
\hline (10) & 2010 & $\mathrm{P}$ & Prox & $\begin{array}{l}\text { ILUMA ultra cone } \\
\text { beam CT scanner }\end{array}$ & $\begin{array}{l}\text { Trophy Trex } \\
\text { X-ray unit, } \\
\text { Progeny } \\
\text { Vision DX, } \\
\text { Digora } \\
\text { Optime, }\end{array}$ & $\begin{array}{l}\text { No significant } \\
\text { difference between } \\
\text { the systems. }\end{array}$ \\
\hline
\end{tabular}


Table 1(Continued). Summary of Studies on the Diagnostic Accuracy of CBCT in the Detection of Dental Caries

\begin{tabular}{|c|c|c|c|c|c|c|}
\hline \multirow[b]{2}{*}{$\begin{array}{l}\text { Author } \\
\text { reference }\end{array}$} & \multirow[b]{2}{*}{ Year } & \multicolumn{2}{|c|}{ Caries } & \multicolumn{2}{|c|}{ Modality } & \multirow[b]{2}{*}{ Results } \\
\hline & & $\begin{array}{c}\text { Type } \\
\text { of } \\
\text { teeth }\end{array}$ & $\begin{array}{c}\text { Surface } \\
\text { Occ / } \\
\text { Prox }\end{array}$ & $C B C T$ & Intraoral & \\
\hline (11) & 2011 & $\mathrm{P}$ & Prox & $\begin{array}{l}\text { NewTom 9000; } \\
\text { Accuitomo } \\
\text { 3DX; Kodak } \\
\text { 9000 3D; } \\
\text { ProMax 3D; and } \\
\text { DCT PRO }\end{array}$ & & $\begin{array}{l}\text { No significant } \\
\text { difference between } \\
\text { systems. }\end{array}$ \\
\hline (12) & 2011 & $\mathrm{P}$ & Both & $\begin{array}{l}\text { Kodak } 9500 \\
\text { Cone Beam 3D } \\
\text { System }\end{array}$ & $\begin{array}{l}\text { Trophy } \\
\text { ETX } \\
\text { intraoral X- } \\
\text { ray unit and } \\
\text { Digora } \\
\text { Optime }\end{array}$ & $\begin{array}{l}\text { Significant } \\
\text { difference seen } \\
\text { between CBCT and } \\
\text { conventional } \\
\text { radiography for } \\
\text { occlusal caries. } \\
\text { No significant } \\
\text { difference between } \\
\text { systems for } \\
\text { proximal caries. }\end{array}$ \\
\hline (13) & 2011 & $\mathrm{P}$ & Prox & $\begin{array}{l}\text { Pax-500ECT, } \\
\text { ProMax 3D }\end{array}$ & $\begin{array}{l}\text { F-speed } \\
\text { films } \\
\text { (Insight } \\
\text { Dental } \\
\text { Film) }\end{array}$ & $\begin{array}{l}\text { CBCT fared better than } \\
\text { intraoral radiography in } \\
\text { detecting secondary } \\
\text { caries. }\end{array}$ \\
\hline (14) & 2013 & $\mathrm{P}$ & Prox & $\begin{array}{l}\text { 3D Accuitomo } \\
\text { FPD80 }\end{array}$ & $\begin{array}{l}\text { Digora } \\
\text { Toto } \\
\text { Digora } \\
\text { Optime }\end{array}$ & $\begin{array}{l}\text { CBCT fared better } \\
\text { than intraoral } \\
\text { systems in } \\
\text { detecting } \\
\text { approximal carious } \\
\text { lesions }\end{array}$ \\
\hline$(15)$ & 2014 & $\mathrm{P}$ & Prox & Kodak 9000 3D & & $\begin{array}{l}\text { CBCT was more } \\
\text { accurate in } \\
\text { detecting cavitation } \\
\text { in proximal } \\
\text { surfaces than } \\
\text { bitewing } \\
\text { radiographs }\end{array}$ \\
\hline
\end{tabular}


Many authors ${ }^{(6,10-12)}$ have indicated that CBCT systems offer no significant improvement on the diagnosis of dental caries when compared with intraoral radiographic techniques. However, some authors ${ }^{(5,9,14,15)}$ have reported greater caries detection using CBCT systems than intraoral radiography. Only Charuakkra, et al., ${ }^{(13)}$ reported $\mathrm{CBCT}$ to be better than film-based bitewing techniques at diagnosing artificially created, secondary caries under proximal, radiopaque restorations. No studies in the current literature have explored the potentially deleterious effect of HDO, particularly coronal metallic dental restorations, on dental caries diagnosis.

Extensive review of the English literature indicates that no author has yet quantified the effect of artifact production by coronal HDO (e.g. dental amalgam) in CBCT images. The purpose of this investigation is to help characterize image quality degredation of beam hardening and scatter introduced on adjacent and regional dental tooth structures when HDOs are introduced into CBCT images. This should provide a theoretical basis for further studies on the effect of HDO's on the detection of coronal dental caries diagnosis on CBCT images with the presence of dental amalgam in the dental arch. 


\section{CHAPTER II}

\section{HYPOTHESES}

\section{Objectives}

The aims of this research are:

- To develop an in vitro anatomic model representative of the human dental arches incorporating extracted human teeth with bone and soft tissue simulation material corresponding to human voxel gray values $(\mathrm{GV})$ on images.

- To develop a sequence of HDO configurations within the in vitro model of the dental arches representing increasing local and overall artifact effects to simulate various clinical scenarios.

- To quantify the effect of artifacts on local and peripheral noise on axial images obtained on 3 different CBCT systems by measuring the variability of gray value (GV) on homogeneous test cylinders acting as controls at standard positions using a non-proprietary, readily available medical/dental image software program.

- To quantify the effect of the following independent variables on the local and peripheral noise (dependent variable) on images obtained on 3 different CBCT systems:

- Inter-arch position (Maxilla/Mandible)

- Number of HDO 
- Intra-arch position (Location of HDO relative to controls)

- Variations in acquisition settings $(\mathrm{kV}$, nominal resolution, exposure time and number of basis images)

\section{Null Hypothesis}

It is hypothesized that:

- There are no effects on image quality on CBCT images with the introduction of HDO (i.e. dental amalgam).

- The effects on image quality on CBCT images with the introduction of HDO does not depend upon the inter-arch position (Maxilla/Mandible) of HDOs.

- The effects on image quality on CBCT images with the introduction of HDO does not depend upon intra-arch number of HDOs.

- The effects on image quality on CBCT images with the introduction of HDO does not depend upon intra-arch proximity (unilateral, anterior or contralateral) of HDOs.

- The effects on image quality on CBCT images with the introduction of HDO does not depend upon $\mathrm{CBCT}$ acquisition parameters such as $\mathrm{kV}$, nomimal resolution, exposure time and number of basis projections. 


\section{CHAPTER III}

\section{MATERIALS AND METHODS}

\section{Overall Research Design:}

This in vitro laboratory-based study was designed to provide a quantitative analysis of the effects of CBCT image artifacts produced by high density objects (HDOs) such as metallic restorations on image quality using a simulated model of the dental arches imaged at various acquisition parameters with three (3) CBCT systems. Image quality was measured as the percentage difference between gray values $(\Delta \mathrm{GV} \%)$ on a fiducial test cylinder of homogeneous radiodensity in a control and treatment phantom. Fiducial cylinders were positioned at three representative tooth sites (right second premolar, central incisor, and left second premolar) within the dental arches (maxilla/mandible) with fifteen (15) configurations of HDOs using three (3) CBCT units operated at several exposure parameters:

1. 3D Accuitomo 170; J Morita MFG. Corp, Kyoto, Japan (Accuitomo 170)

2. iCAT Next generation; iCAT model 17-19, Imaging Sciences

International Inc., Hatfield, PA, USA. (iCAT NG)

3. Carestream 9000 3D, Carestream/KODAK, Atlanta, GA. (CS 9000) 


\section{In vitro Simulated Dental Arch Model}

Maxillary and mandibular dental arch models were fabricated to simulate the dentition aligned in the average human dental arch form. Initially a life size template was created based on the average shape of human dental arches ${ }^{(16)}$ using utility wax. An impression of the wax models was then taken using alginate (Jeltrate, Dentsply Caulk, Milford, Del.). During pour with type III dental stone (Quickstone, Whip Mix Corporation, Louisville, KY, USA), utility wax was inserted as a substitute for alveolar bone proper to enable insertion of extracted teeth within the reproduced dental arches. Dental stone mixed with plastic round pellets (Freeplastic, Daicel Craft ltd., Tokyo) simulated the radio density of bone ${ }^{(2)}$ and bone marrow in the jaws respectively. Permanent, non-carious, unrestored, sterilized, extracted teeth were used to construct a maxillary and mandibular dental arch. Three uniform test cylinders (average size of a premolar; $25 \mathrm{~mm}$ [height] x 5mm [diameter]) made of type V jade stone (Whip Mix Corporation Louisville, KY, USA) replaced the right central incisor, right and left second premolars in each dental arch. These provided control fiducial objects at three locations with uniform, homogeneously dense material with mean density gray values (GV) approximating dentin. Water was mixed with jade stone in the ratio of $15.5 \mathrm{ml} / 70 \mathrm{~g}$ to achieve a radiodensity similar to that of dentin as measured on a subset of CBCT patient scans (iCAT NG) with no HDOs (Table 2). Measurements were obtained by exporting the DICOM (Digital Image and Communication in Medicine) data and importing it into a non-proprietary image analysis software (OSIRIX MD; Osirix Foundation, Geneva, Switzerland). Gray values of dentin in the same locations as in Table 2, for control models, scanned by Accuitomo 170 and Carestream 9000 3D are given in Table 3. 
Table 2. Comparison of gray values of dentin in the control model (without HDO) and patient radiographs (without HDO) on iCAT Next Generation CBCT Unit (120 kV / 5 $\mathrm{mA})$.

\begin{tabular}{|c|c|c|c|c|c|c|c|c|c|c|c|}
\hline \multirow[t]{3}{*}{ Patient } & \multirow{3}{*}{ Age (yrs) } & \multirow[t]{3}{*}{ Res } & \multirow{3}{*}{$\begin{array}{c}\text { No. of } \\
\text { basis } \\
\text { images }\end{array}$} & \multicolumn{4}{|c|}{ Maxillary } & \multicolumn{4}{|c|}{ Mandibular } \\
\hline & & & & \multicolumn{2}{|l|}{ PM1 } & \multicolumn{2}{|l|}{ M1 } & \multicolumn{2}{|l|}{ PM1 } & \multicolumn{2}{|l|}{ M1 } \\
\hline & & & & $\mathbf{R}$ & $\mathbf{L}$ & $\mathbf{R}$ & $\mathbf{L}$ & $\mathbf{R}$ & $\mathbf{L}$ & $\mathbf{R}$ & $\mathbf{L}$ \\
\hline \multicolumn{2}{|c|}{ Control } & 0. & 200 & 986 & 1076 & 1117 & 1118 & 1220 & 1196 & 1029 & 1009 \\
\hline \multicolumn{2}{|c|}{ Control } & 0.25 & 325 & 970 & 1026 & 871 & 968 & 1135 & 1169 & 966 & 969 \\
\hline 1 & 20 & 0.3 & 576 & 878 & 878 & 779 & 835 & 827 & 985 & 707 & 993 \\
\hline 2 & 16 & 0.3 & 576 & 930 & 965 & 1037 & 907 & 857 & 990 & 1075 & 879 \\
\hline 3 & 14 & 0.3 & 576 & 1740 & 1798 & 1658 & 1793 & 1553 & 1482 & 1658 & 1573 \\
\hline 4 & 16 & 0.3 & 576 & 1015 & 940 & 1131 & 1058 & 1158 & 874 & 1181 & 958 \\
\hline 5 & 19 & 0.3 & 576 & 866 & 908 & 787 & 775 & 739 & 881 & 827 & 833 \\
\hline \multicolumn{4}{|c|}{ MEAN GV for Patients } & 1085 & 1038 & 1053 & 1056 & 1124 & 1057 & 1026 & 1040 \\
\hline
\end{tabular}

*Res, resolution; PM1, First Premolar;M1, First Molar.

Table 3. Gray values of dentin on control model on Accuitomo 170 and Carestream 9000 $3 \mathrm{D}$.

\begin{tabular}{|c|c|c|c|c|c|c|c|c|c|c|c|c|c|}
\hline \multirow[t]{3}{*}{$\begin{array}{c}\text { Pati } \\
\text { ent }\end{array}$} & \multirow[t]{3}{*}{ CBCT } & \multirow[t]{3}{*}{$\begin{array}{l}\boldsymbol{k} \\
\boldsymbol{V} \\
\end{array}$} & \multirow[t]{3}{*}{$\begin{array}{l}m \\
A\end{array}$} & \multirow[t]{3}{*}{ Res } & \multirow{3}{*}{$\begin{array}{c}\text { No. } \\
\text { of } \\
\text { basis } \\
\text { imag } \\
\text { es }\end{array}$} & \multicolumn{4}{|c|}{ Maxillary } & \multicolumn{4}{|c|}{ Mandibular } \\
\hline & & & & & & \multicolumn{2}{|c|}{ PM1 } & \multicolumn{2}{|c|}{ M1 } & \multicolumn{2}{|c|}{ PM1 } & \multicolumn{2}{|c|}{ M1 } \\
\hline & & & & & & $\mathbf{R}$ & $\mathbf{L}$ & $\mathbf{R}$ & $\mathbf{L}$ & $\mathbf{R}$ & $\mathbf{L}$ & $\mathbf{R}$ & $\mathbf{L}$ \\
\hline \multirow[t]{8}{*}{$\begin{array}{l}\text { Cont } \\
\text {-rol }\end{array}$} & Acc & 90 & 5 & 0.25 & 1008 & 1497 & 1381 & 1487 & 1457 & 1384 & 1555 & 1397 & 1384 \\
\hline & Acc & 75 & 5 & 0.25 & 1007 & 1524 & 1376 & 1332 & 1226 & 1597 & 1602 & 1323 & 1276 \\
\hline & Acc & 90 & 5 & 0.25 & 584 & 1254 & 1439 & 1258 & 1279 & 1377 & 1408 & 1542 & 1439 \\
\hline & Acc & 75 & 5 & 0.25 & 584 & 1567 & 1731 & 1526 & 1531 & 1611 & 1619 & 1775 & 1758 \\
\hline & CS 3D & 90 & 10 & 0.2 & 360 & 1357 & 1536 & 1308 & 1495 & 1432 & 1573 & 1394 & 1326 \\
\hline & CS 3D & 75 & 10 & 0.2 & 360 & 1408 & 1429 & 1468 & 1424 & 1465 & 1579 & 1379 & 1310 \\
\hline & CS 3D & 90 & 10 & 0.08 & 360 & 1451 & 1580 & 1470 & 1667 & 1210 & 1260 & 1323 & 1477 \\
\hline & CS 3D & 75 & 10 & 0.08 & 360 & 1385 & 1454 & 1301 & 1616 & 1311 & 1305 & 1468 & 1304 \\
\hline
\end{tabular}

*Res, resolution; PM1, First Premolar; M1, First Molar. 
The models were imaged multiple times at various exposure parameters with removal and substitution of specific teeth with comparable teeth prepared with standard, mesial-occlusal-distal (MOD) dental amalgam coronally restored premolars and molars. Insertion of the restored teeth was performed in a specific configuration representing an increasing level of coronal high density objects (HDO). The entire model was immersed in water to simulate soft tissue attenuation within the head. After many samples were tried, the most appropriate container [ 8 " (height) x 6.5 " (diameter) with water filled at the 4.5" mark], which fit the model and could hold enough water to simulate attenuation within the head, was used. Images of the dental arches with unrestored teeth without coronal HDO at each exposure parameter were used as controls.

\section{High Density Object (HDO) Configuration}

Dental amalgam restored teeth were arranged in fifteen (15) configurations to reproduce imaging scenarios of increasing unilateral and bilateral coronal HDO in the dental arches (Table: 4) (Appendix A).

\section{Cone Beam Computed Tomographic Imaging}

Three (3) CBCT systems were used to image the maxillary and mandibular models together. For each system, the dental arch models with no HDOs present were imaged at each exposure setting (control). Then the models with HDOs according to the fifteen (15) configurations previously described (six unilateral and nine bilateral) were imaged. The total number of scans performed for each unit was equal to the number of exposure settings used (y) times the HDO configurations used (15). 
Table 4. Location of High Density Objects

\begin{tabular}{|c|c|c|c|c|}
\hline \multirow[b]{2}{*}{ Config. } & \multirow{2}{*}{$\begin{array}{l}\text { Relative to } \\
\text { the Dental } \\
\text { Arch }\end{array}$} & \multirow[b]{2}{*}{ Relative to the Test Cylinder } & \multicolumn{2}{|c|}{ Specific Configuration of $\mathrm{HDO}$} \\
\hline & & & Right & Left \\
\hline 1 & Unilateral & Adjacent to test cylinder & PM1 & - \\
\hline 5 & & & M1 \& M2 & - \\
\hline 9 & & & $\begin{array}{l}\text { M1 \& M2 \& } \\
\quad \text { M3 }\end{array}$ & - \\
\hline 2 & Unilateral & One tooth away from test cylinder & M2 & - \\
\hline 6 & & & M2 \& M3 & - \\
\hline 3 & Unilateral & Two teeth away from test cylinder & M3 & - \\
\hline 12 & Bilateral & Adjacent to test cylinder & PM1 & PM1 \\
\hline 15 & & & M1 & M1 \& M2 \\
\hline 16 & & & M1 & $\mathrm{M} 1, \mathrm{M} 2 \& \mathrm{M} 3$ \\
\hline 18 & & & M1 \& M2 & M1 \& M2 \\
\hline 19 & & & M1 \& M2 & M1, M2 \& M3 \\
\hline 21 & & & $\mathrm{M} 1, \mathrm{M} 2 \& \mathrm{M} 3$ & M1, M2 \&M 3 \\
\hline 13 & Bilateral & One tooth away from test cylinder & M2 & M2 \\
\hline 24 & & & M2 \& M3 & M2 \& M3 \\
\hline 25 & Bilateral & Two teeth away from test cylinder & M3 & M3 \\
\hline \multicolumn{5}{|c|}{$\begin{array}{l}\text { Config.: Configuration; PM1:First Premolar; M1: First Molar; M2: Second Molar; M3: Third } \\
\text { Molar }\end{array}$} \\
\hline
\end{tabular}

The exposure parameters used with the Carestream 9000 3D (Carestream Health, Atlanta, GA) CBCT unit are shown in Table 4. The model was attached on the base provided by the manufacturer and oriented in the center of FOV, with the occlusal plane parallel to the horizontal plane using laser orientation beams. As this CBCT system has a limited FOV, images were taken separately for three regions (Anterior, left and right posterior) at the exposure parameters shown in Table 5. Axial slices at the default nominal resolution were exported as uncompressed, multi-file images in DICOM format from the proprietary software (KODAK Dental Imaging Software 6.11.7.0, Carestream 
Health Inc.,2007, Atlanta, GA) and imported into OsiriX MD software (OsiriX Foundation, Geneva, Switzerland) for analysis.

Table 5. Exposure parameters for the Carestream 9000 3D CBCT System

\begin{tabular}{cccccc}
\hline $\boldsymbol{k} \boldsymbol{V}$ & $\boldsymbol{m A}$ & $\begin{array}{c}\text { Nominal Resolution } \\
(\mathbf{m m})\end{array}$ & Mode & Time $(\boldsymbol{s})$ & $\begin{array}{c}\text { FOV }(\text { diameter } \boldsymbol{x} \\
\text { height })(\boldsymbol{c m})\end{array}$ \\
\hline 90 & 10 & 0.2 & - & $10.62^{*}$ & $5 * 3.7$ \\
90 & 10 & 0.076 & - & $10.59^{\wedge}$ & $5 * 3.7$ \\
75 & 10 & 0.2 & - & 10.62 & $5 * 3.7$ \\
75 & 10 & 0.076 & - & 10.62 & $5 * 3.7$ \\
\hline$* 360$ basis images & & & &
\end{tabular}

2) iCAT Next Generation System

The exposure parameters used with the iCAT (Next Generation, Imaging Sciences International, Hatsfield, PA) are shown in Table 6. The model was placed on the base provided by the manufacturer, in the center of FOV and aligned with the horizontal plane using the laser light system for orientation. Scout images were taken prior to scanning to ensure correct placement of the model. Axial slices at the default nominal resolution were exported as uncompressed, multi-file images in DICOM format from the proprietary software (i-CATVision $\mathrm{Q}^{\mathrm{TM}}$ software (Imaging Sciences International, Hatsfield, PA) and imported into OsiriX MD software for analysis.

Table 6. Exposure parameters for the iCAT Next Generation CBCT System

\begin{tabular}{cccccc}
\hline $\boldsymbol{k V}$ & $\boldsymbol{m A}$ & $\begin{array}{c}\text { Nominal Resolution } \\
(\boldsymbol{m m})\end{array}$ & Mode & Time $(\mathbf{s})$ & $\begin{array}{c}\text { FOV (diameter } \boldsymbol{x} \text { height }) \\
(\mathbf{c m})\end{array}$ \\
\hline 120 & 5 & 0.4 & - & $4.8^{*}$ & $160 * 80$ \\
120 & 5 & 0.25 & - & $26.9^{\wedge}$ & $160 * 80$ \\
\hline
\end{tabular}

*160 Basis images; ^ 619 Basis images 


\section{3) Accuitomo 170}

The exposure parameters used with the 3D Accuitomo 170 (J. Morita, Kyoto, Japan) CBCT system is shown in Table 7. Using boxes as support, models were elevated to enable alignment within the field of view (FOV) using the laser positioning lights such that the occlusal plane was parallel to the horizontal plane and models were in the center of the field of view (FOV). Scout images were taken to confirm the correct positioning of the model within the FOV. Default $0.25 \mathrm{~mm}$ axial images were exported as uncompressed multi-file images in Digital Imaging and Communications in Medicine (DICOM) format from the proprietary software (iDixel image processing software, J. Morita USA, Inc, Irvine, USA) and imported into OsiriX MD software for analysis.

Table 7. Exposure parameters for the Acciutomo 170 CBCT System

\begin{tabular}{cccccc}
\hline $\boldsymbol{k V}$ & $\boldsymbol{m A}$ & Nominal Resolution $(\boldsymbol{m m})$ & Mode & Time $(\mathbf{s})$ & $\begin{array}{c}\text { FOV (diameter } \boldsymbol{x} \text { height }) \\
(\mathbf{c m})\end{array}$ \\
\hline 90 & 5 & 0.25 & Standard* & 17.5 & $140 * 100$ \\
90 & 5 & 0.25 & Hi-fi^ & 30.8 & $140 * 100$ \\
75 & 5 & 0.25 & Standard* & 17.5 & $140 * 100$ \\
75 & 5 & 0.25 & Hi-fi\# & 30.8 & $140 * 100$ \\
\hline *584 Basis images; ${ }^{\wedge}$ 1008 Basis images; \#1007 Basis images
\end{tabular}




\section{Analysis}

\section{CBCT Image Evaluation}

All images were imported into OsiriX MD (OsiriX Foundation, Geneva, Switzerland) to avoid any disparity in measurements produced by using unit specific proprietary CBCT system softwares. Osirix MD was chosen for this evaluation because, unlike other softwares that were tried, this software was able to uncompress and open DICOM files from all the CBCT systems that were used.

X-ray attenuation, in terms of mean grey value $(\mathrm{GV})$, was measured at six levels relative to the occlusal plane vertically on each test cylinder: at the occlusal plane $(0 \mathrm{~mm})$, $2 \mathrm{~mm}, 4 \mathrm{~mm}, 10 \mathrm{~mm}, 15 \mathrm{~mm}$, and $25 \mathrm{~mm}$. The level on each cylinder was measured from the occlusal surface of amalgam restorations. To facilitate reproducibility, the density region of interest (ROI) was customized and fixed at $0.175 \mathrm{~cm}^{2}$ and used for every measurement. A square of an area of $0.175 \mathrm{~cm}^{2}$ was found to be the largest area that fit within the circumference of the test cylinder. The same protocol was used for all experimental and control models.

\section{Data Analysis}

Benic, et al., ${ }^{(2)}$ used a methodology to study the effect of beam hardening around implants by comparing mean GV values circumferentially around implants. This methodology was adapted for use in this research. Mean GV measured within the defined ROI on control models for each scanning parameter was designated as $\mathrm{GV}_{\text {control. }}$. Differences of gray values $(\Delta \mathrm{GV})$ between models with $\left(\mathrm{GV}_{\text {Test }}\right)$ and without $\left(\mathrm{GV}_{\text {control }}\right)$ HDO were calculated as percentages using the following formula: 


$$
\Delta G V \%=\left[\left(G V_{\text {Test }}-G V_{\text {control }}\right) / G V_{\text {control }}\right] * 1000^{(2)}
$$

Graphically, mean GV\% was plotted overall for each test cylinder (ipsilateral, center, contralateral) and again according to adjacent and non-adjacent groups of configurations against the independent variables. Each CBCT system has its own confounding variables that make statistical comparison between the systems, using the results from this experimental method, biased. Therefore for each CBCT system, the GV\% values were analyzed statistically by the General Linear model by comparing mean values in a MANOVA design against all independent variables with HDO present unilaterally and bilaterally (Table 4) and at different parameters (Table 5,6,7) on the same CBCT system. 


\section{CHAPTER IV}

\section{RESULTS}

\section{Overview}

Two approaches were used to analyze and present the data:

1) To visualize and describe trends for each CBCT unit, descriptive statistics were provided by plotting two graphs:

a. An overall graph of $\Delta \mathrm{GV} \%$ (y-axis) for each test cylinder location (Center, contralateral and, ipsilateral) according to each independent variable and,

b. A second graph stratifying these results according to position of the HDO relative to the test cylinder (adjacent vs. non-adjacent).

2) Statistical comparison to compare the effects of the categorized independent variables. The multiple categorical analysis for parametric data is ANOVA. However, application of ANOVA assumes that the data conforms to two assumptions:

a. The data is normally distributed (Poisson distribution), and

b. They data have equal variances (Ranges / s.d. are similar).

Prior to performing the analysis, grouped independent variables were analyzed to determine Normality (Anderson-Darling test for Normality) and Equal variances (F-test for normally distributed data and Levene's Test for non-normally distributed data). Because overall the data for $\Delta \mathrm{GV} \%$ were not normally 
distributed nor had equal variances (Table 8), the general linear model was chosen.

\section{1) Descriptive}

For each $\mathrm{CBCT}$ unit two plots are generated. The assumptions are that $\triangle \mathrm{GV} \%$ is an index of image quality. Interpretation of the values for $\Delta \mathrm{GV} \%$ was as follows:

- A $\Delta \mathrm{GV} \%$ close to zero (0) means that there is little or no difference in image quality between the phantom with no HDOs (control) and the phantom with HDOs (test).

- A $-\Delta \mathrm{GV} \%$ depicts a relative hyper-density or dark region associated with beam hardening and,

- $\mathrm{A}+\Delta \mathrm{GV} \%$ is a relative hypo-density or light region associated with scattered radiation.

The first figure shows the overall plot of $\Delta \mathrm{GV} \%$ (y-axis) for each test cylinder location (center, contralateral and, ipsilateral) according to each independent variable. The second plot shows the plot of $\Delta \mathrm{GV} \%$ (y-axis) according to position of the HDO relative to the test cylinder (adjacent vs. non-adjacent). 
Table 8. Choosing the Appropriate Multiple Comparison Test - Normality (AndersonDarling test) And Equal Variance Tests for independent variables for each CBCT unit

\begin{tabular}{|c|c|c|c|c|c|c|c|c|}
\hline \multirow[b]{2}{*}{$\begin{array}{l}C B C T \\
\text { Unit }\end{array}$} & \multirow{2}{*}{$\begin{array}{l}\text { Independen } \\
t \text { Variable }\end{array}$} & \multicolumn{3}{|c|}{ Normality } & \multicolumn{4}{|c|}{ Equal Variances* } \\
\hline & & $\mathbf{A}$ & $\mathbf{N}$ & Sig. & $\begin{array}{l}\text { Bartlet } \\
\text { t's Test }\end{array}$ & Sig. $\dagger$ & $\begin{array}{l}\text { Levene' } \\
\text { s Test }\end{array}$ & Sig. $\dagger$ \\
\hline \multirow[t]{9}{*}{ CS 9000} & & 11.039 & 2160 & $<0.005$ & & & & \\
\hline & Config. & & & & 201.45 & 0.000 & 8.92 & 0.000 \\
\hline & Depth & & & & 308.33 & 0.000 & 45.05 & 0.000 \\
\hline & Arch & & & & 0.94 & 0.288 & 0.07 & 0.798 \\
\hline & Location & & & & 133.53 & 0.000 & 37.44 & 0.000 \\
\hline & $\mathrm{kV}$ & & & & 1.04 & 0.514 & 0.78 & 0.376 \\
\hline & Resolution & & & & 2.20 & 0.000 & 76.27 & 0.000 \\
\hline & Sidedness & & & & 2.19 & 0.000 & 104.64 & 0.000 \\
\hline & Proximity & & & & 1.30 & 0.000 & 8.69 & 0.003 \\
\hline \multirow[t]{8}{*}{ iCAT NG } & & 180.70 & 1080 & $<0.005$ & & & & \\
\hline & Config. & & & & 3024.8 & 0.000 & 38.11 & 0.000 \\
\hline & Depth & & & & 20.93 & 0.001 & 1.90 & 0.091 \\
\hline & Arch & & & & 1.32 & 0.001 & 1.83 & 0.176 \\
\hline & Location & & & & 14.51 & 0.001 & 2.69 & 0.069 \\
\hline & Resolution & & & & 0.84 & 0.049 & 0.52 & 0.471 \\
\hline & Sidedness & & & & 372.10 & 0.000 & 295.05 & 0.000 \\
\hline & Proximity & & & & 307.07 & 0.000 & 282.77 & 0.000 \\
\hline \multirow[t]{9}{*}{$\begin{array}{l}\text { Accuitom } \\
\text { o } 170\end{array}$} & & 498.74 & 2160 & $<0.005$ & & & & \\
\hline & Config. & & & & 4695.1 & 0.000 & 85.58 & 0.000 \\
\hline & Depth & & & & 71.09 & 0.000 & 1.44 & 0.206 \\
\hline & Arch & & & & 1.42 & 0.000 & 5.53 & 0.019 \\
\hline & Location & & & & 60.54 & 0.000 & 5.45 & 0.004 \\
\hline & $\mathrm{kV}$ & & & & 0.78 & 0.000 & 0.53 & 0.466 \\
\hline & Frames & & & & 1.03 & 0.628 & 0.12 & 0.732 \\
\hline & Sidedness & & & & 33.15 & 0.000 & 231.30 & 0.000 \\
\hline & Proximity & & & & 46.88 & 0.000 & 229.33 & 0.000 \\
\hline
\end{tabular}

Sig., statistical significance; Config., Configuration

* The use of F-Test or Levene's Test depends on the normality of the data. If the data is normally distributed then the F-Value should be used. If the data is not normally distributed then the Levene's test should be used.

$\digamma$ If $p>0.05$, the variances can be assumed to be roughly the same and ANOVA assumptions are valid. 
Figure 1. CS 9000 interval plot showing $\Delta \mathrm{GV} \%$ for each test cylinder location according to each independent variable (Depth, $\mathrm{kV}$ and resolution)

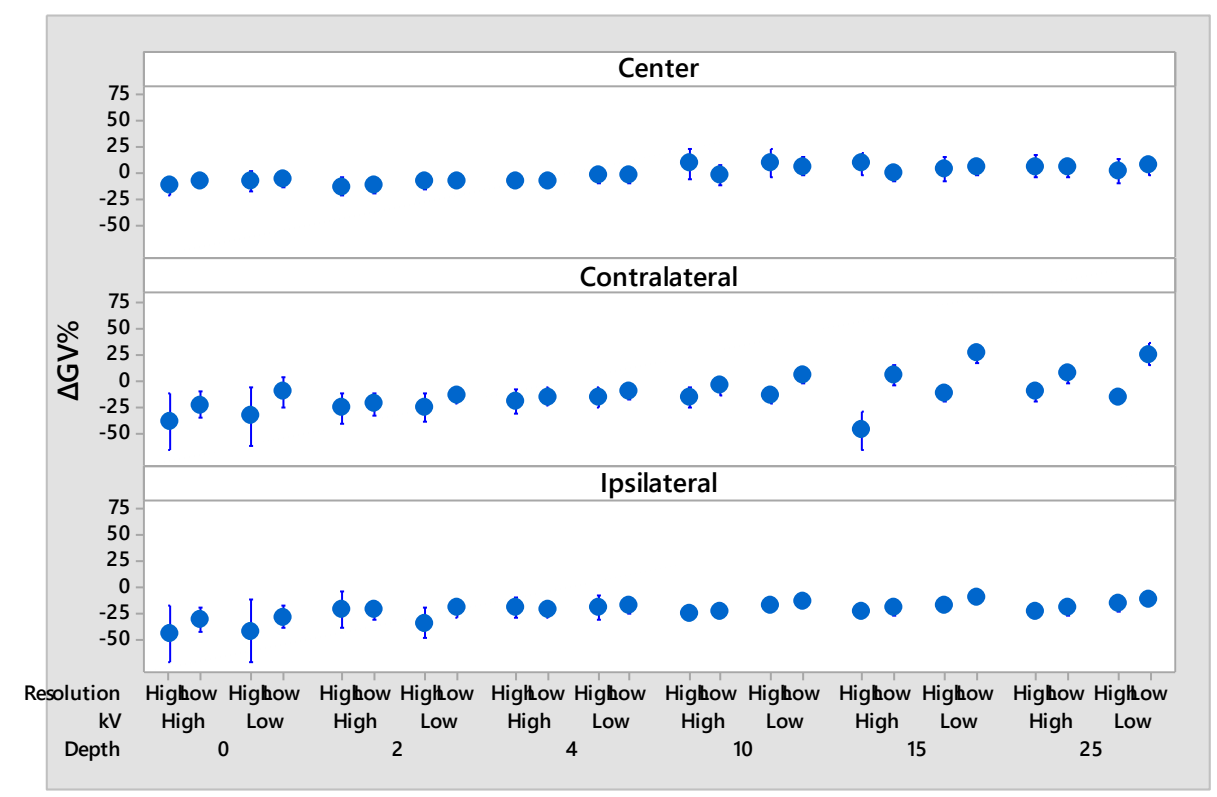

Figure 1 shows the overall plot of $\Delta \mathrm{GV} \%$ (y-axis) for the CS 9000D. As a trend, overall image quality $(\Delta \mathrm{GV} \%)$ :

- Is reduced overall due to the effects of HDO by increasing the overall density of the image due to beam hardening $(-\Delta \mathrm{GV} \%)$,

- Is worse for ipsilateral cylinders followed by contralateral followed by anteriorly placed cylinders,

- Is better with lower resolution $(0.2 \mathrm{~mm})$ than higher resolution $(0.076 \mathrm{~mm})$ particularly for contralateral and ipsilateral cylinders,

- Appears to be independent of $\mathrm{kV}$,

- Improves with increasing distance from the occlusal plane, especially within $2 \mathrm{~mm}$ to $4 \mathrm{~mm}$.

- Appears to be independent of arch (maxillary/mandibular). 
Figure 2. CS 9000 interval plot showing $\Delta \mathrm{GV} \%$ for each test cylinder location according to each independent variable (Depth, $\mathrm{kV}$ and resolution) according to position of the HDO relative to the test cylinder (adjacent vs. non-adjacent).

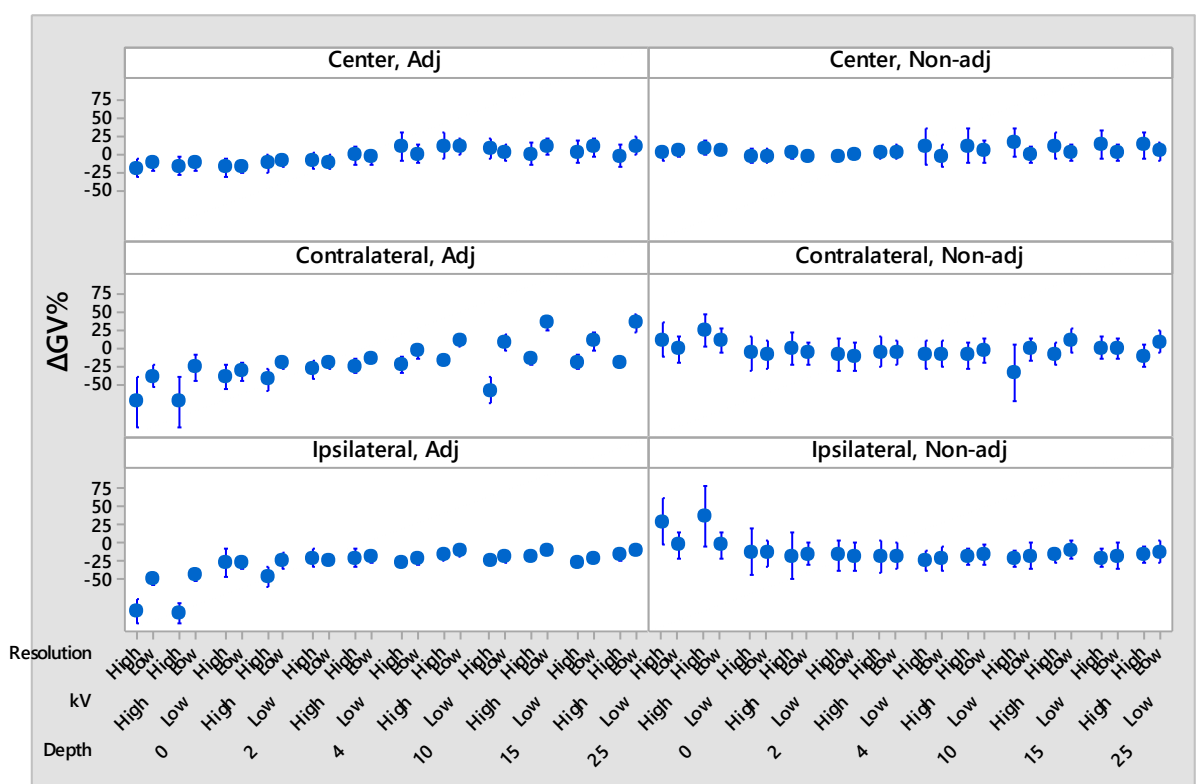

Figure 2 shows a plot of $\Delta \mathrm{GV} \%$ (y-axis) according to position of the HDO relative to the test cylinder (adjacent vs. non-adjacent) for the CS 9000D. As a trend, image quality $(\Delta \mathrm{GV} \%)$

- Is independent of the position of the HDO in the anterior region.

- Is worse on both the ipsilateral and contralateral side when the HDO is adjacent the test cylinder,

- Is worse for high resolution than low resolution for both contralateral and ipsilateral sides when the HDO is adjacent to the cylinder,

- Beam hardening effects are reduced $(-\Delta \mathrm{GV} \%)$ and scattering effects become more prominent $(\Delta \mathrm{GV} \%$ becomes more positive) for both contralateral and ipsilateral sides when the HDO is non-adjacent to the cylinders; this appears to be more pronounced for high resolution than low resolution,

- Appears to be independent of $\mathrm{kV}$, irrespective of position of the HDO, 
- Improves with increasing distance from the occlusal plane, with the greatest change occurring from $2 \mathrm{~mm}$ to $4 \mathrm{~mm}$.

\section{iCAT Next Generation}

Figure 3. iCAT NG interval plot showing $\Delta \mathrm{GV} \%$ for each test cylinder location according to each independent variable (Depth, $\mathrm{kV}$ and resolution).

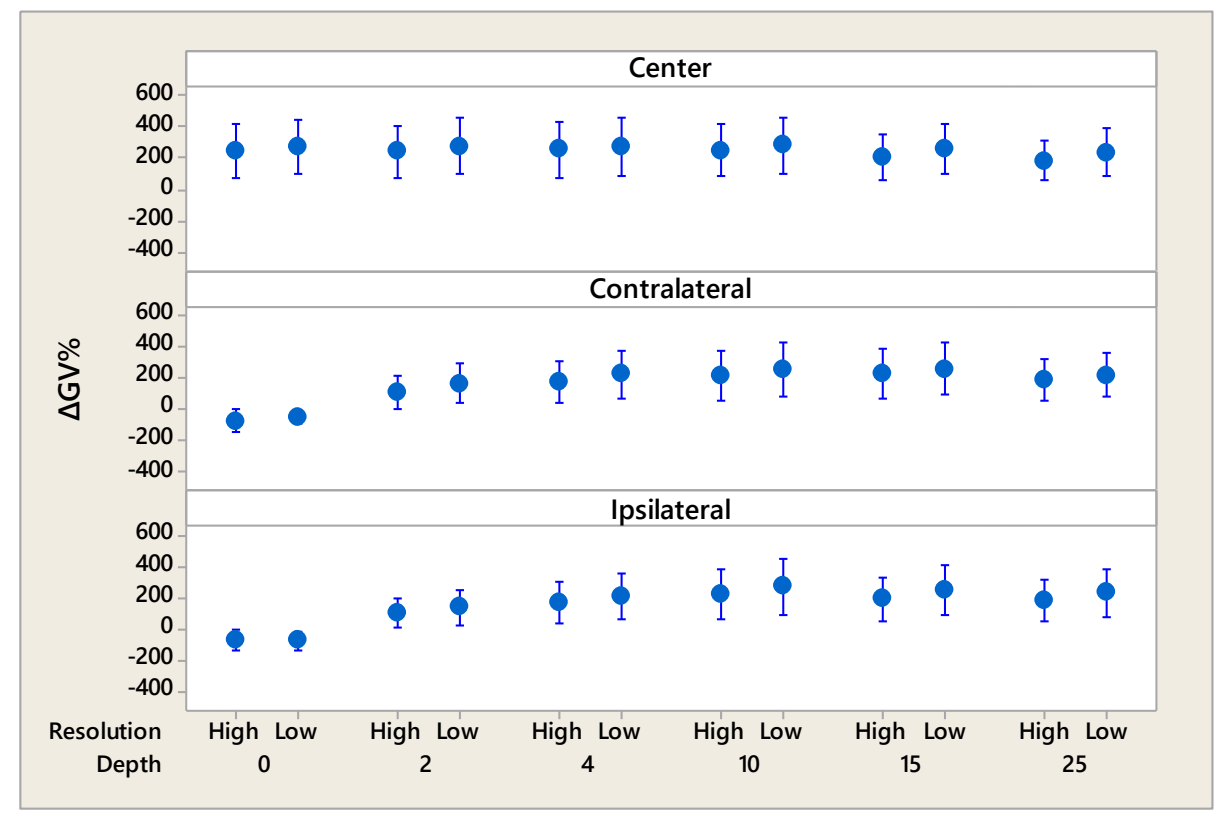

Figure 3 shows the overall plot of $\Delta \mathrm{GV} \%$ (y-axis) for the iCAT NG. As a trend, overall image quality $(\Delta \mathrm{GV} \%)$ :

- Is reduced due to scatter $(+\Delta \mathrm{GV} \%)$ for anterior cylinders similarly at all heights relative to the occlusal plane,

- Is reduced due to beam hardening $(-\Delta \mathrm{GV} \%)$ at the level of the occlusal plane for both ipsilateral and contralateral cylinders,

- Is reduced due to scatter is $(+\Delta \mathrm{GV} \%)$ at levels below the occlusal plane for both ipsilateral and contralateral cylinders reaching a plateau at about $10 \mathrm{~mm}$,

- Is reduced with lower resolution $(0.2 \mathrm{~mm})$ than higher resolution $(0.076 \mathrm{~mm})$, 
particularly for contralateral and ipsilateral cylinders.

Figure 4. iCAT NG interval plot showing $\Delta \mathrm{GV} \%$ for each test cylinder location according to each independent variable (Depth, $\mathrm{kV}$ and resolution) according to position of the HDO relative to the test cylinder (adjacent vs. non-adjacent).

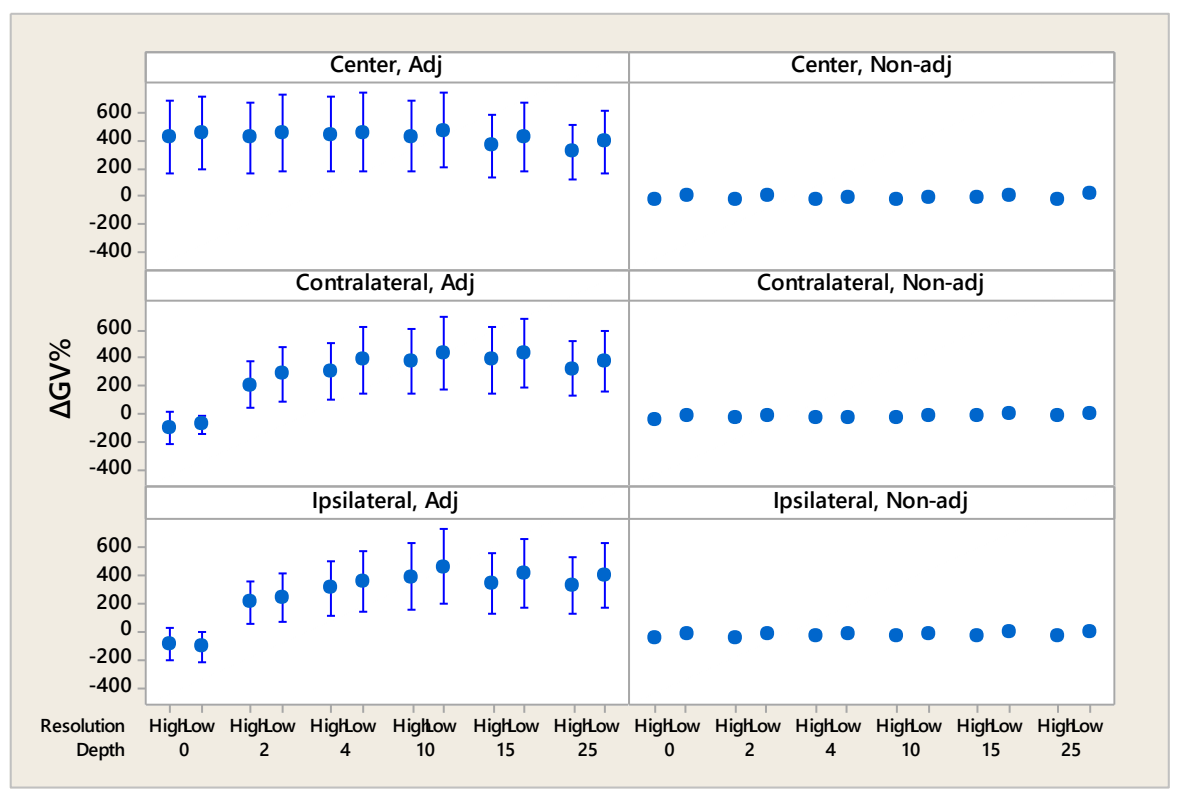

Figure 4 shows a plot of $\Delta \mathrm{GV} \%$ (y-axis) according to position of the HDO relative to the test cylinder (adjacent vs. non-adjacent) for the iCAT NG. As a trend, image quality $(\Delta \mathrm{GV} \%)$ :

- Is minimally affected or marginally reduced due to beam hardening $(-\Delta \mathrm{GV} \%)$ when HDOs are non-adjacent to the test cylinder at all positions (center, contr- and ipsilateral),

- Follows similar trends as described above when HDOs are adjacent the test cylinder. 


\section{D Accuitomo 170}

Figure 5. 3D Accuitomo 170 interval plot showing $\Delta \mathrm{GV} \%$ for each test cylinder location according to each independent variable (Depth, $\mathrm{kV}$ and resolution).

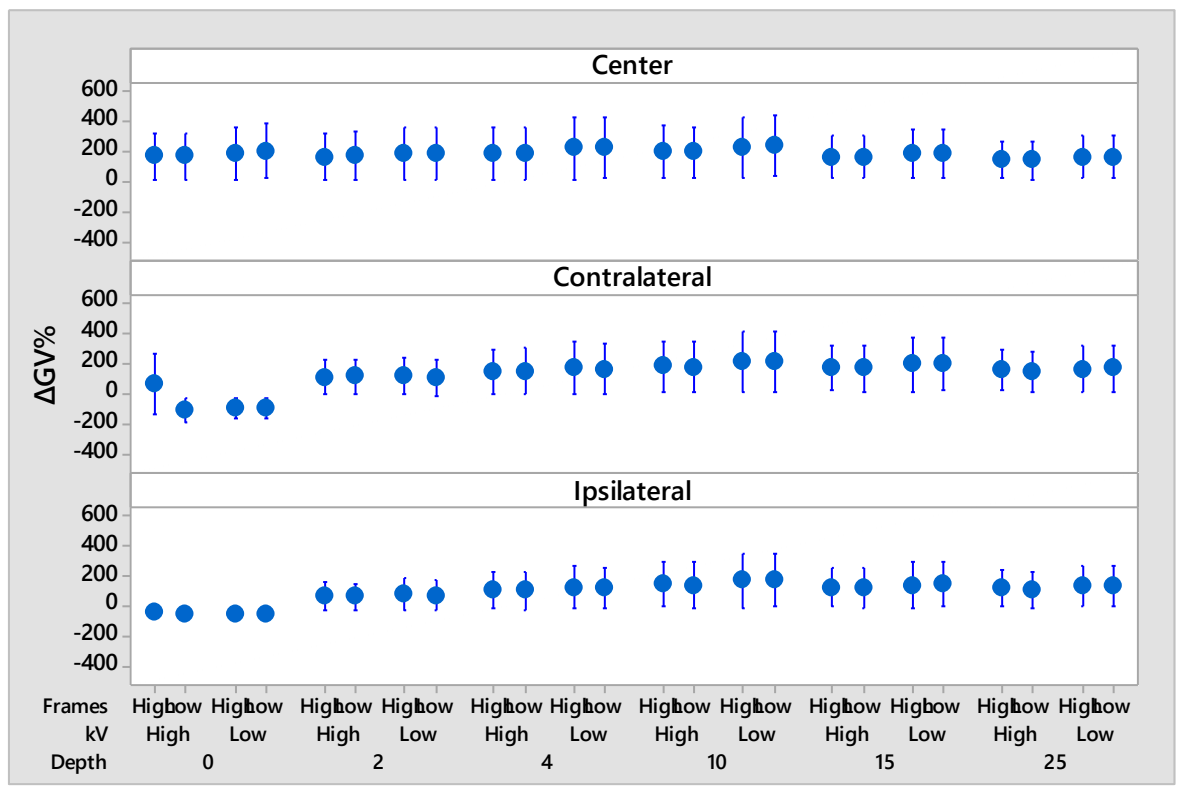

Figure 5 shows the overall plot of $\Delta \mathrm{GV} \%$ (y-axis) for the 3D Accuuitomo 170. As a trend, overall image quality $(\Delta \mathrm{GV} \%)$ :

- Is reduced due to scatter $(+\Delta \mathrm{GV} \%)$ for anterior cylinders similarly at all heights relative to the occlusal plane:

- Is reduced due to beam hardening $(-\Delta G V \%)$ at the level of the occlusal plane for both ipsilateral and contralateral cylinders,

- Is reduced due to scatter is $(+\Delta \mathrm{GV} \%)$ at levels below the occlusal plane for both ipsilateral and contralateral cylinders reaching a plateau at about $4 \mathrm{~mm}$,

- Appears to be independent of $\mathrm{kV}$ or number of projection frames. 
Figure 6. 3D Accuitomo 170 interval plot showing $\Delta \mathrm{GV} \%$ for each test cylinder location according to each independent variable (Depth, $\mathrm{kV}$ and resolution) according to position of the HDO relative to the test cylinder (adjacent vs. non-adjacent).

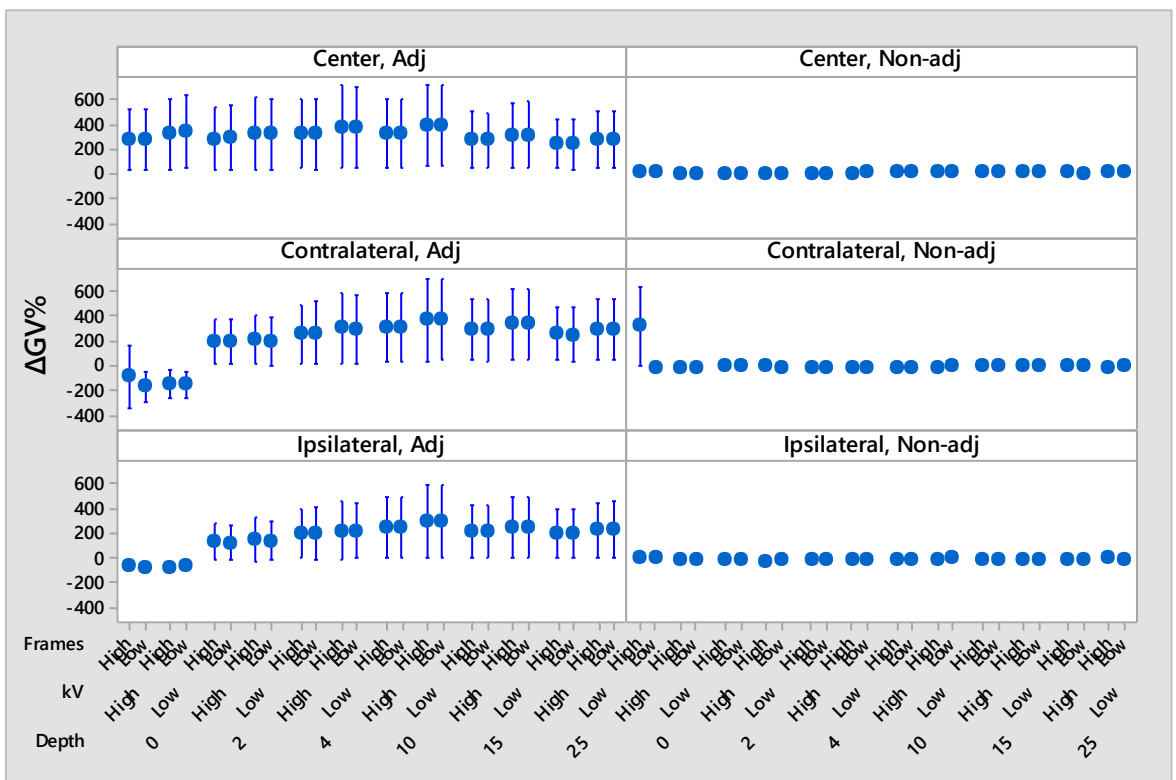

Figure 6 shows a plot of $\Delta \mathrm{GV} \%$ (y-axis) according to position of the HDO relative to the test cylinder (adjacent vs. non-adjacent) for the 3D Accuuitomo 170. As a trend, image quality $(\Delta \mathrm{GV} \%)$ :

- Is highly dependent on the position of the HDO in that non-adjacent HDOs minimally affect image quality for anterior, ipsi- and contra-lateral sides. 


\section{2) Analytical}

\section{Multiple Analysis of Variance for Each CBCT Unit}

Tables 9-31 shows the General Linear Model for each CBCT unit for the dependent variable, $\Delta \mathrm{GV} \%$, grouped according to each independent variable.

\section{CS $90003 D$}

Table 9 shows that there is no overall effect from the arch type (maxilla mean $\Delta \mathrm{GV} \%$ $=12.7$, mandible mean $\Delta \mathrm{GV} \%=11.8 ; \mathrm{T}=-0.7072, \mathrm{p}=0.48$ ) or sidedness (unilateral, bilateral). Therefore data from the maxillary and mandibular models can be combined and the effect of sidedness and arch ignored. Of the total variability, $76 \%$ is unexplained (Error SS). However, of the significant factors the most important, in order, are configuration (10.4\%), arch location (6.6\%), level of cylinder $(4.7 \%)$, proximity $(4.1 \%)$, resolution $(1.6 \%)$ and, $\mathrm{kV}(0.7 \%)$.

Table 9. CS 90003D GLM

\begin{tabular}{llllll}
\hline Source & $\boldsymbol{D F}$ & $\boldsymbol{S S}$ & $\boldsymbol{M S}$ & $\boldsymbol{F}$ & Sig. \\
\hline Configuration & $\mathbf{1 4}$ & 253284 & 18092 & 20.96 & 0.000 \\
Level of Cylinder & $\mathbf{5}$ & 112844 & 22569 & 26.15 & 0.000 \\
Arch & $\mathbf{1}$ & 432 & 432 & 0.50 & 0.480 \\
Arch location & $\mathbf{2}$ & 159866 & 79933 & 92.60 & 0.000 \\
kV & $\mathbf{1}$ & 16853 & 16853 & 19.53 & 0.000 \\
Resolution & $\mathbf{1}$ & 38211 & 38211 & 44.27 & 0.000 \\
Sidedness & $\mathbf{1}$ & 737 & 737 & 0.69 & 0.408 \\
Proximity & $\mathbf{1}$ & 99030 & 99030 & 92.12 & 0.000 \\
Error & $\mathbf{2 1 3 5}$ & 1842868 & 863 & & \\
Total & $\mathbf{2 1 5 9}$ & $\mathbf{2 4 2 4 3 5 8}$ & & & \\
\hline
\end{tabular}

GLM, General Linear Model; DF, degrees of freedom; SS, sum of the squares; MS, mean square; $F, F$ value; Sig., statistical significance. 


\section{Post Hoc Analysis}

Table 10 shows Post Hoc pairwise comparison for $\Delta \mathrm{GV} \%$ at various configurations of HDO artifacts. The analysis indicates that overall there are no differences between specific artifact configurations within overlapping groups of increasing $\Delta \mathrm{GV} \%$ :

- Group (Configuration 12, 1, 15, 19 and 16) [Range; $\Delta \mathrm{GV} \%, 29.61$ to 18.8],

- Group (Configuration 1, 15, 19, 16, 24, 18, 21, 5, 2 and 3) [Range; $\Delta \mathrm{GV} \%, 19.94$ to 11.48],

- Group (Configuration 19, 16, 24, 18, 21, 5, 2 and 3) [Range; $\Delta \mathrm{GV} \%, 18.98$ to 7.96],

- Group (Configuration 24, 18, 21, 5, 2, 3 and 9) [Range; $\Delta \mathrm{GV} \%, 17.32$ to 6.59],

- Group (Configuration 5, 2, 3, 9 and 25) [Range; $\Delta \mathrm{GV} \%, 14.51$ to 3.12],

- Group (Configuration 2, 3, 9, 25 and 6) [Range; $\Delta \mathrm{GV} \%, 11.48$ to 0.27].

- Configuration $13(\Delta \mathrm{GV} \%, 17.83)$ is different from all other configurations.

Identifying trends within this series taking into account the proximity of the test cylinder to the HDO, whether the HDO was uni- or bilateral and number of HDOs indicates that configurations with the worst $\Delta \mathrm{GV} \%$ are those test cylinders adjacent to the HDO or configurations that have the highest number of HDOs overall. 
Table 10. Post Hoc pairwise comparison (Tukey) for $\Delta \mathrm{GV} \%$ for various configurations of HDO Artifact

\begin{tabular}{|c|c|c|c|c|c|}
\hline \multicolumn{3}{|l|}{$\begin{array}{l}\text { Pairwise } \\
\text { Comparison }\end{array}$} & \multicolumn{3}{|c|}{ Analysis } \\
\hline Baseline & M.ean & Level & Mean & T-value & p \\
\hline \multirow[t]{14}{*}{ Config. 1} & -19.94 & Config. 5 & -14.51 & 1.567 & 0.9656 \\
\hline & & Config. 9 & -6.59 & 3.855 & 0.0099 \\
\hline & & Config. 2 & -11.48 & 2.443 & 0.4820 \\
\hline & & Config. 6 & -0.27 & 5.681 & 0.0000 \\
\hline & & Config. 3 & -7.96 & 3.46 & 0.0401 \\
\hline & & Config. 12 & -29.61 & -2.794 & 0.2498 \\
\hline & & Config. 15 & -19.91 & 0.009 & 1.0000 \\
\hline & & Config. 16 & -18.8 & 0.33 & 1.0000 \\
\hline & & Config. 18 & -17.28 & 0.766 & 1.0000 \\
\hline & & Config. 19 & -18.98 & 0.276 & 1.0000 \\
\hline & & Config. 21 & -15.82 & 1.189 & 0.9976 \\
\hline & & Config. 13 & 17.83 & -10.907 & 0.0000 \\
\hline & & Config. 24 & -17.32 & 0.756 & 1.0000 \\
\hline & & Config. 25 & -3.12 & 4.858 & 0.0002 \\
\hline \multirow[t]{13}{*}{ Config. 5} & -14.51 & Config. 9 & -6.59 & 2.288 & 0.5999 \\
\hline & & Config. 2 & -11.48 & -0.876 & 0.9999 \\
\hline & & Config. 6 & -0.27 & 4.114 & 0.0036 \\
\hline & & Config. 3 & -7.96 & -1.892 & 0.8574 \\
\hline & & Config. 12 & -29.61 & 4.361 & 0.0013 \\
\hline & & Config. 15 & -19.91 & 1.5584 & 0.9672 \\
\hline & & Config. 16 & -18.8 & 1.23793 & 0.9963 \\
\hline & & Config. 18 & -17.28 & 0.8010 & 1.0000 \\
\hline & & Config. 19 & -18.98 & 1.2914 & 0.9943 \\
\hline & & Config. 21 & -15.82 & 0.3787 & 1.0000 \\
\hline & & Config. 13 & 17.83 & -9.34 & 0.0000 \\
\hline & & Config. 24 & -17.32 & 0.8119 & 1.0000 \\
\hline & & Config. 25 & -3.12 & -3.291 & 0.0683 \\
\hline \multirow[t]{12}{*}{ Config. 9} & 6.59 & Config. 2 & -11.48 & 1.412 & 0.9865 \\
\hline & & Config. 6 & -0.27 & -1.826 & 0.8878 \\
\hline & & Config. 3 & -7.96 & 0.395 & 1.0000 \\
\hline & & Config. 12 & -29.61 & 6.649 & 0.0000 \\
\hline & & Config. 15 & -19.91 & 3.8460 & 0.0103 \\
\hline & & Config. 16 & -18.8 & 3.52550 & 0.0323 \\
\hline & & Config. 18 & -17.28 & 3.0886 & 0.1215 \\
\hline & & Config. 19 & -18.98 & 3.5790 & 0.0270 \\
\hline & & Config. 21 & -15.82 & 2.6662 & 0.3258 \\
\hline & & Config. 13 & 17.83 & -7.05 & 0.0000 \\
\hline & & Config. 24 & -17.32 & 3.0995 & 0.1179 \\
\hline & & Config. 25 & -3.12 & -1.003 & 0.9996 \\
\hline
\end{tabular}


Table 10 (continued). Post Hoc pairwise comparison (Tukey) for $\Delta \mathrm{GV} \%$ for various configurations of artifact

\begin{tabular}{|c|c|c|c|c|c|}
\hline \multicolumn{3}{|l|}{$\begin{array}{l}\text { Pairwise } \\
\text { Comparison }\end{array}$} & \multicolumn{3}{|c|}{ Analysis } \\
\hline Baseline & Mean & Level & Mean & T-value & $\mathbf{P}$ \\
\hline \multirow[t]{11}{*}{ Config. 2} & 11.48 & Config. 6 & -0.27 & 3.238 & 0.0798 \\
\hline & & Config. 3 & -7.96 & 1.016 & 0.9996 \\
\hline & & Config. 12 & -29.61 & 5.237 & 0.0000 \\
\hline & & Config. 15 & -19.91 & 2.4345 & 0.4888 \\
\hline & & Config. 16 & -18.8 & 2.11396 & 0.7258 \\
\hline & & Config. 18 & -17.28 & 1.6771 & 0.9403 \\
\hline & & Config. 19 & -18.98 & 2.1675 & 0.6885 \\
\hline & & Config. 21 & -15.82 & -1.255 & 0.9958 \\
\hline & & Config. 13 & 17.83 & -8.46 & 0.0000 \\
\hline & & Config. 24 & -17.32 & -1.688 & 0.9372 \\
\hline & & Config. 25 & -3.12 & 2.415 & 0.5037 \\
\hline \multirow[t]{10}{*}{ Config. 6} & 0.27 & Config. 3 & -7.96 & 2.221 & 0.6493 \\
\hline & & Config. 12 & -29.61 & 8.475 & 0.0000 \\
\hline & & Config. 15 & -19.91 & 5.6724 & 0.0000 \\
\hline & & Config. 16 & -18.8 & 5.35187 & 0.0000 \\
\hline & & Config. 18 & -17.28 & 4.9150 & 0.0001 \\
\hline & & Config. 19 & -18.98 & 5.4054 & 0.0000 \\
\hline & & Config. 21 & -15.82 & 4.4926 & 0.0007 \\
\hline & & Config. 13 & 17.83 & -5.23 & 0.0001 \\
\hline & & Config. 24 & -17.32 & 4.9259 & 0.0001 \\
\hline & & Config. 25 & -3.12 & -0.823 & 1.0000 \\
\hline \multirow[t]{9}{*}{ Config. 3} & 7.96 & Config. 12 & -29.61 & 6.254 & 0.0000 \\
\hline & & Config. 15 & -19.91 & 3.4509 & 0.0413 \\
\hline & & Config. 16 & -18.8 & 3.13042 & 0.1084 \\
\hline & & Config. 18 & -17.28 & 2.6935 & 0.3086 \\
\hline & & Config. 19 & -18.98 & 3.1839 & 0.0933 \\
\hline & & Config. 21 & -15.82 & 2.2711 & 0.6123 \\
\hline & & Config. 13 & 17.83 & -7.45 & 0.0000 \\
\hline & & Config. 24 & -17.32 & 2.7044 & 0.3019 \\
\hline & & Config. 25 & -3.12 & -1.398 & 0.9877 \\
\hline \multirow[t]{8}{*}{ Config. 12} & 29.61 & Config. 15 & -19.91 & 2.803 & 0.2449 \\
\hline & & Config. 16 & -18.8 & 3.124 & 0.1105 \\
\hline & & Config. 18 & -17.28 & 3.560 & 0.0287 \\
\hline & & Config. 19 & -18.98 & 3.070 & 0.1277 \\
\hline & & Config. 21 & -15.82 & 3.983 & 0.0061 \\
\hline & & Config. 13 & 17.83 & -13.701 & 0.0000 \\
\hline & & Config. 24 & -17.32 & 3.549 & 0.0298 \\
\hline & & Config. 25 & -3.12 & 7.652 & 0.0000 \\
\hline
\end{tabular}


Table 10 (continued). Post Hoc pairwise comparison (Tukey) for $\Delta \mathrm{GV} \%$ for various configurations of artifact

\begin{tabular}{|c|c|c|c|c|c|}
\hline Pairwise & & & & Analysis & \\
\hline Baseline & Mean & Level & Mean & T-value & $\mathbf{p}$ \\
\hline Config. 15 & 19.91 & Config. 16 & -18.8 & 0.3205 & 1.0000 \\
\hline & & Config. 18 & -17.28 & 0.7574 & 1.0000 \\
\hline & & Config. 19 & -18.98 & 0.2670 & 1.0000 \\
\hline & & Config. 21 & -15.82 & 1.1798 & 0.9978 \\
\hline & & Config. 13 & 17.83 & -10.90 & 0.0000 \\
\hline & & Config. 24 & -17.32 & 0.7465 & 1.0000 \\
\hline & & Config. 25 & -3.12 & 4.8491 & 0.0002 \\
\hline Config. 16 & -18.8 & Config. 18 & -17.28 & 0.43688 & 1.0000 \\
\hline & & Config. 19 & -18.98 & -0.05350 & 1.0000 \\
\hline & & Config. 21 & -15.82 & 0.85927 & 0.9999 \\
\hline & & Config. 13 & 17.83 & -10.58 & 0.0000 \\
\hline & & Config. 24 & -17.32 & 0.42600 & 1.0000 \\
\hline & & Config. 25 & -3.12 & 4.52858 & 0.0006 \\
\hline Config. 18 & -17.28 & Config. 19 & -18.98 & -0.4904 & 1.0000 \\
\hline & & Config. 21 & -15.82 & 0.4224 & 1.0000 \\
\hline & & Config. 13 & 17.83 & -10.14 & 0.0000 \\
\hline & & Config. 24 & -17.32 & -0.0109 & 1.0000 \\
\hline & & Config. 25 & -3.12 & 4.0917 & 0.0039 \\
\hline Config. 19 & -18.98 & Config. 21 & -15.82 & 0.9128 & 0.9999 \\
\hline & & Config. 13 & 17.83 & -10.63 & 0.0000 \\
\hline & & Config. 24 & -17.32 & 0.4795 & 1.0000 \\
\hline & & Config. 25 & -3.12 & 4.5821 & 0.0005 \\
\hline Config. 21 & -15.82 & Config. 13 & 17.83 & 9.72 & 0.0000 \\
\hline & & Config. 24 & -17.32 & -0.4333 & 1.0000 \\
\hline & & Config. 25 & -3.12 & 3.6693 & 0.0197 \\
\hline Config. 13 & -17.83 & Config. 24 & -17.32 & -10.15 & 0.0000 \\
\hline & & Config. 25 & -3.12 & -6.05 & 0.0000 \\
\hline Config. 24 & -17.32 & Config. 25 & -3.12 & 4.1026 & 0.0037 \\
\hline
\end{tabular}

Config., configuration 
Table 11 shows the Post Hoc pairwise comparison (Tukey) for $\Delta \mathrm{GV} \%$ at various levels of the cylinder. At the occlusal level $(0 \mathrm{~mm})$, image quality $(\Delta \mathrm{GV} \%)$ is significantly worse than $4 \mathrm{~mm}$ or greater at this level. At $2 \mathrm{~mm}$ from the occlusal level (2mm), HDOs significantly affect $\Delta \mathrm{GV} \%$ values $10 \mathrm{~mm}$ and above. At $4 \mathrm{~mm}$ from the occlusal level (4mm), image quality $(\Delta \mathrm{GV} \%)$ is significantly worse than $15 \mathrm{~mm}$ and above. At the $10 \mathrm{~mm}$ level from the occlusal level $(10 \mathrm{~mm})$, there is no difference between artifacts at the $4 \mathrm{~mm}$ level or higher at the 15 and $25 \mathrm{~mm}$ levels.

Table 11. Post Hoc pairwise comparison (Tukey) for $\Delta \mathrm{GV} \%$ at various level of cylinder

\begin{tabular}{llllll}
\hline $\begin{array}{l}\text { Pairwise } \\
\text { Comparison }\end{array}$ & & & & Analysis & \\
\hline Baseline & Mean & Level & Mean & T-value & p \\
\hline Occlusal (0mm) & -23.85 & 2 & -18.99 & 2.218 & 0.229 \\
& & 4 & -13.3 & 4.817 & 0.000 \\
& & 10 & -7.13 & 7.635 & 0.000 \\
& & 15 & -6.66 & 7.846 & 0.000 \\
$2 \mathrm{~mm}$ & 25 & -3.59 & 9.251 & 0.000 \\
& -18.99 & 4 & -13.3 & 2.59 & 0.0975 \\
& & 10 & -7.13 & 5.417 & 0.000 \\
$4 \mathrm{~mm}$ & 15 & -6.66 & 5.627 & 0.000 \\
& -13.3 & 10 & -3.59 & 7.033 & 0.000 \\
& & 15 & -7.13 & 2.818 & 0.0546 \\
$10 \mathrm{~mm}$ & & 25 & -6.66 & 3.029 & 0.0296 \\
& & 15 & -3.59 & 4.434 & 0.000 \\
$15 \mathrm{~mm}$ & -7.13 & 25 & -6.66 & 0.21 & 0.999 \\
& & 25 & -3.59 & 1.616 & 0.5878 \\
& -6.66 & -3.59 & 1.406 & 0.7237 \\
\hline
\end{tabular}


Table 12 shows the Post Hoc pairwise comparison for $\Delta \mathrm{GV} \%$ at various arch locations. The $\Delta \mathrm{GV} \%$ at different arch locations are all significantly different from each other with minimal overall loss in image quality in the center, more on the contralateral side and the greatest in the ipsilateral side.

Table 12. Post Hoc pairwise comparison (Tukey) for $\Delta \mathrm{GV} \%$ at various arch locations

\begin{tabular}{llllll}
\hline $\begin{array}{l}\text { Pairwise } \\
\text { Comparison }\end{array}$ & & & \multicolumn{3}{c}{ Analysis } \\
\hline Baseline & Mean & Level & Mean & T-value & P \\
\hline Center & -1.18 & Ipsilateral & -22.16 & -13.55 & 0.000 \\
& & Contralateral & -13.4 & -7.9 & 0.000 \\
Ipsilateral & -22.16 & Contralateral & -13.4 & -5.65 & 0.000 \\
\hline
\end{tabular}

Table 13 shows Post Hoc pairwise comparison for $\Delta \mathrm{GV} \%$ at two kVs. Overall, images performed at $90 \mathrm{kV}$ produce a significantly worse image quality than at $75 \mathrm{kV}$.

Table 13. Post Hoc pairwise comparison (Tukey) for $\Delta \mathrm{GV} \%$ at two $\mathrm{kV}$ levels.

\begin{tabular}{llllll}
\hline $\begin{array}{l}\text { Pairwise } \\
\text { Comparison }\end{array}$ & & & \multicolumn{2}{c}{ Analysis } & \\
\hline Baseline & Mean & Level & Mean & T-value & p \\
\hline $75 \mathrm{kV}$ & -9.46 & $90 \mathrm{kV}$ & -15.04 & 4.419 & 0.000 \\
\hline
\end{tabular}

Table 14 shows the Post Hoc pairwise comparison for $\Delta \mathrm{GV} \%$ at various resolutions. Overall images obtained at higher resolution $(0.076 \mathrm{~mm})$ provide a greater loss in image quality than at low resolution $(0.2 \mathrm{~mm})$.

Table 14. Post Hoc pairwise comparison (Tukey) for $\Delta \mathrm{GV} \%$ at high and low resolutions.

\begin{tabular}{llllll}
\hline $\begin{array}{l}\text { Pairwise } \\
\text { Comparison }\end{array}$ & & & & \multicolumn{2}{l}{ Analysis } \\
\hline Baseline & Mean & Level & Mean & T-Value & P \\
\hline Hi Res & -16.46 & Low Res & -8.05 & 6.65 & 0.000 \\
\hline
\end{tabular}

Table 15 shows the Post Hoc pairwise comparison for $\Delta \mathrm{GV} \%$ according to 
whether HDO are uni- or bilaterally positioned and shows that image quality is independent of position of HDO.

Table 15. Post Hoc pairwise comparison (Tukey) for $\Delta \mathrm{GV} \%$ according to the side where HDO is positioned.

\begin{tabular}{llllll}
\hline $\begin{array}{l}\text { Pairwise } \\
\text { Comparison }\end{array}$ & & & & \multicolumn{3}{c}{ Analysis } & \\
\hline Baseline & Mean & Level & Mean & T-Value & P \\
\hline Unilateral & -10.12 & Bilateral & -11.33 & 0.8277 & 0.4078 \\
\hline
\end{tabular}

Table 16 shows Post Hoc pairwise comparison for $\Delta \mathrm{GV} \%$ at position of $\mathrm{HDO}$ relative to test cylinder. Overall images with HDOs positioned adjacent to test cylinder show significantly greater loss in image quality than images with HDOs non-adjacent to test cylinder.

Table16. Post Hoc pairwise comparison (Tukey) for $\Delta \mathrm{GV} \%$ at position of HDMO relative to test cylinder

\begin{tabular}{llllll}
\hline $\begin{array}{l}\text { Pairwise } \\
\text { Comparison }\end{array}$ & & & & Analysis \\
\hline Baseline & Mean & Level & Mean & T-Value & P \\
\hline Non-adjacent & -3.72 & Adjacent & -17.74 & 9.598 & 0.000 \\
\hline
\end{tabular}




\section{iCAT Next Generation}

Table 17 shows that all factors significantly affect image quality $(\Delta G V \%)$. Of the total variability, $20.9 \%$ is unexplained (Error SS). The most important factors, in order, are configuration $(74.6 \%)$, proximity $(12.9 \%)$, sidedness $(12.1 \%)$, level of cylinder $(3 \%)$, arch location $(1.1 \%)$, resolution $(0.2 \%)$, and finally arch $(0.18 \%)$.

Table 17. Analysis of Variance for iCAT Next Generation

\begin{tabular}{ccllll}
\hline Source & $\boldsymbol{D F}$ & \multicolumn{1}{c}{$\boldsymbol{S S}$} & \multicolumn{1}{c}{$\boldsymbol{M S}$} & \multicolumn{1}{c}{$\boldsymbol{F}$} & Sig. \\
\hline Configuration & $\mathbf{1 4}$ & 127092974 & 9078070 & 268.87 & 0.000 \\
Level of Cylinder & $\mathbf{5}$ & 5181565 & 1036313 & 30.69 & 0.000 \\
Arch & $\mathbf{1}$ & 314102 & 314102 & 9.30 & 0.002 \\
Arch location & $\mathbf{2}$ & 1829191 & 914596 & 27.09 & 0.000 \\
Resolution & $\mathbf{1}$ & 378076 & 378076 & 11.20 & 0.001 \\
Sidedness & $\mathbf{1}$ & 20590531 & 20590531 & 185.89 & 0.000 \\
Proximity & $\mathbf{1}$ & 22039565 & 22039565 & 198.97 & 0.000 \\
Error & $\mathbf{1 0 5 6}$ & 35654947 & 33764 & & \\
Total & $\mathbf{1 0 7 9}$ & $\mathbf{1 7 0 4 5 0 8 5 5}$ & & & \\
\hline
\end{tabular}

GLM, General Linear Model; DF, degrees of freedom; SS, sum of the squares; MS, mean square; $F, F$ value; Sig., statistical significance. 


\section{Post Hoc Analysis}

Table 18 shows Post Hoc pairwise comparison for $\Delta \mathrm{GV} \%$ at various configurations of HDOs. Overall there are differences between specific artifacts configurations within 3 groups of increasing $\Delta \mathrm{GV} \%$ :

- Group (Configuration 24, 21, 6, 12, 3, 1, 5, 13, 2, 25 and 9) [Range $\Delta \mathrm{GV} \%,-49.31$ to $-1.14]$

- Group (Configuration 18) [ $\Delta \mathrm{GV} \%, 639.96]$,

- Group (Configuration 19, 15 and 16) [Range $\Delta \mathrm{GV} \%, 775.59$ to 810.90].

Identifying trends within this series, taking into consideration proximity of the test cylinder to the HDMO, whether the HDMO was uni- or bilateral and number of HDMOs indicates that configurations with the worst $\Delta \mathrm{GV} \%$ are those test cylinders adjacent to the HDO or that have the highest number of HDOs overall. 
Table 18. Post Hoc pairwise comparison (Tukey) for $\Delta \mathrm{GV} \%$ for various configurations of HDOs

\begin{tabular}{|c|c|c|c|c|c|}
\hline \multicolumn{3}{|l|}{$\begin{array}{l}\text { Pairwise } \\
\text { Comparison }\end{array}$} & \multicolumn{3}{|c|}{ Analysis } \\
\hline Baseline & Mean & Level & Mean & T-value & $\mathbf{P}$ \\
\hline \multirow[t]{14}{*}{ Config. 1} & 16.68 & Config. 5 & -15.94 & 0.024 & 1.0000 \\
\hline & & Config. 9 & -1.14 & 0.507 & 1.0000 \\
\hline & & Config. 2 & -6.26 & 0.340 & 1.0000 \\
\hline & & Config. 6 & -21.75 & -0.166 & 1.0000 \\
\hline & & Config. 3 & -19.18 & -0.082 & 1.0000 \\
\hline & & Config. 12 & -21.21 & -0.148 & 1.0000 \\
\hline & & Config. 15 & 786.66 & 26.231 & 0.0000 \\
\hline & & Config. 16 & 810.90 & 27.023 & 0.0000 \\
\hline & & Config. 18 & 639.96 & 21.441 & 0.0000 \\
\hline & & Config. 19 & 775.59 & 25.870 & 0.0000 \\
\hline & & Config. 21 & -33.67 & -0.555 & 1.0000 \\
\hline & & Config. 13 & -9.99 & 0.218 & 1.0000 \\
\hline & & Config. 24 & -49.31 & -1.066 & 0.9993 \\
\hline & & Config. 25 & -4.11 & 0.410 & 1.0000 \\
\hline \multirow[t]{13}{*}{ Config. 5} & -15.94 & Config. 9 & -1.14 & 0.4833 & 1.0000 \\
\hline & & Config. 2 & -6.26 & -0.316 & 1.0000 \\
\hline & & Config. 6 & -21.75 & -0.1897 & 1.0000 \\
\hline & & Config. 3 & -19.18 & 0.10569 & 1.0000 \\
\hline & & Config. 12 & -21.21 & 0.1722 & 1.0000 \\
\hline & & Config. 15 & 786.66 & -26.21 & 0.0000 \\
\hline & & Config. 16 & 810.90 & -27.00 & 0.0000 \\
\hline & & Config. 18 & 639.96 & -21.42 & 0.0000 \\
\hline & & Config. 19 & 775.59 & -25.85 & 0.0000 \\
\hline & & Config. 21 & -33.67 & 0.5790 & 1.0000 \\
\hline & & Config. 13 & -9.99 & -0.194 & 1.0000 \\
\hline & & Config. 24 & -49.31 & 1.0897 & 0.9991 \\
\hline & & Config. 25 & -4.11 & -0.3862 & 1.0000 \\
\hline \multirow[t]{11}{*}{ Config. 9} & -1.14 & Config. 2 & -6.26 & 0.167 & 1.0000 \\
\hline & & Config. 6 & -21.75 & 0.6730 & 1.0000 \\
\hline & & Config. 3 & -19.18 & 0.58901 & 1.0000 \\
\hline & & Config. 12 & -21.21 & 0.6555 & 1.0000 \\
\hline & & Config. 15 & 786.66 & -25.72 & 0.0000 \\
\hline & & Config. 16 & 810.90 & -26.52 & 0.0000 \\
\hline & & Config. 18 & 639.96 & -20.93 & 0.0000 \\
\hline & & Config. 19 & 775.59 & -25.36 & 0.0000 \\
\hline & & Config. 21 & -33.67 & 1.0623 & 0.9993 \\
\hline & & Config. 13 & -9.99 & 0.289 & 1.0000 \\
\hline & & Config. 24 & -49.31 & 1.5731 & 0.9645 \\
\hline
\end{tabular}


Table 18(Continued). Post Hoc pairwise comparison (Tukey) for $\Delta \mathrm{GV} \%$ for various configurations of HDOs

\begin{tabular}{|c|c|c|c|c|c|}
\hline $\begin{array}{l}\text { Pairwise } \\
\text { Comparison }\end{array}$ & & & & Analysis & \\
\hline Baseline & Mean & Level & Mean & T-value & $\mathbf{P}$ \\
\hline & & Config. 25 & -4.11 & 0.0971 & 1.0000 \\
\hline \multirow[t]{11}{*}{ Config. 2} & -6.26 & Config. 6 & -21.75 & -0.506 & 1.0000 \\
\hline & & Config. 3 & -19.18 & -0.422 & 1.0000 \\
\hline & & Config. 12 & -21.21 & 0.4884 & 1.0000 \\
\hline & & Config. 15 & 786.66 & -25.89 & 0.0000 \\
\hline & & Config. 16 & 810.90 & -26.68 & 0.0000 \\
\hline & & Config. 18 & 639.96 & -21.10 & 0.0000 \\
\hline & & Config. 19 & 775.59 & -25.53 & 0.0000 \\
\hline & & Config. 21 & -33.67 & -0.895 & 0.9999 \\
\hline & & Config. 13 & -9.99 & 0.122 & 1.0000 \\
\hline & & Config. 24 & -49.31 & -1.406 & 0.9870 \\
\hline & & Config. 25 & -4.11 & 0.070 & 1.0000 \\
\hline \multirow[t]{17}{*}{ Config. 6} & -21.75 & Config. 3 & -19.18 & -0.08397 & 1.0000 \\
\hline & & Config. 12 & -21.21 & -0.0175 & 1.0000 \\
\hline & & Config. 15 & 786.66 & -0.384 & 0.0000 \\
\hline & & Config. 16 & 810.90 & -27.19 & 0.0000 \\
\hline & & Config. 18 & 639.96 & -21.61 & 0.0000 \\
\hline & & Config. 19 & 775.59 & -26.04 & 0.0000 \\
\hline & & Config. 21 & -33.67 & -0.5108 & 1.0000 \\
\hline & & Config. 13 & -9.99 & -0.384 & 1.0000 \\
\hline & & Config. 24 & -49.31 & 0.9001 & 0.9999 \\
\hline & & Config. 25 & -4.11 & -0.5759 & 1.0000 \\
\hline & & Config. 16 & 810.90 & -27.10 & 0.0000 \\
\hline & & Config. 18 & 639.96 & -21.52 & 0.0000 \\
\hline & & Config. 19 & 775.59 & -25.95 & 0.0000 \\
\hline & & Config. 21 & -33.67 & 0.4733 & 1.0000 \\
\hline & & Config. 13 & -9.99 & -0.300 & 1.0000 \\
\hline & & Config. 24 & -49.31 & 0.9841 & 0.9997 \\
\hline & & Config. 25 & -4.11 & -0.4919 & 1.000 \\
\hline \multirow[t]{7}{*}{ Config. 12} & -21.21 & Config. 15 & 786.66 & 26.3795 & 0.0000 \\
\hline & & Config. 16 & 810.90 & 27.1712 & 0.0000 \\
\hline & & Config. 18 & 639.96 & 21.5893 & 0.0000 \\
\hline & & Config. 19 & 775.59 & 26.0182 & 0.0000 \\
\hline & & Config. 21 & -33.67 & -0.4068 & 1.0000 \\
\hline & & Config. 13 & -9.99 & 0.3664 & 1.0000 \\
\hline & & Config. 24 & -49.31 & -0.9176 & 0.9999 \\
\hline
\end{tabular}


Table 18(Continued). Post Hoc pairwise comparison (Tukey) for $\Delta \mathrm{GV} \%$ for various configurations of HDOs

\begin{tabular}{|c|c|c|c|c|c|}
\hline \multirow{2}{*}{$\begin{array}{l}\text { Pairwise } \\
\text { Comparison } \\
\text { Baseline } \\
\end{array}$} & \multirow[b]{2}{*}{ Mean } & \multirow[b]{2}{*}{ Level } & \multicolumn{3}{|c|}{ Analysis } \\
\hline & & & Mean & T-value & $\mathbf{P}$ \\
\hline \multirow{8}{*}{ Config. 15} & & Config. 25 & -4.11 & 0.5584 & 1.0000 \\
\hline & 786.66 & Config. 16 & 810.90 & 0.79 & 1.0000 \\
\hline & & Config. 18 & 639.96 & -4.79 & 0.0002 \\
\hline & & Config. 19 & 775.59 & -0.36 & 1.0000 \\
\hline & & Config. 21 & -33.67 & -26.79 & 0.0000 \\
\hline & & Config. 13 & -9.99 & 26.013 & 0.0000 \\
\hline & & Config. 24 & -49.31 & -27.30 & 0.0000 \\
\hline & & Config. 25 & -4.11 & -25.82 & 0.0000 \\
\hline \multirow[t]{6}{*}{ Config. 16} & 810.90 & Config. 18 & 639.96 & -5.58 & 0.0000 \\
\hline & & Config. 19 & 775.59 & -1.15 & 0.9983 \\
\hline & & Config. 21 & -33.67 & -27.58 & 0.0000 \\
\hline & & Config. 13 & -9.99 & 26.805 & 0.0000 \\
\hline & & Config. 24 & -49.31 & -28.09 & 0.0000 \\
\hline & & Config. 25 & -4.11 & -26.61 & 0.0000 \\
\hline \multirow[t]{5}{*}{ Config. 18} & 639.96 & Config. 19 & 775.59 & 4.43 & 0.0009 \\
\hline & & Config. 21 & -33.67 & -22.00 & 0.0000 \\
\hline & & Config. 13 & -9.99 & 21.223 & 0.0000 \\
\hline & & Config. 24 & -49.31 & -22.51 & 0.0000 \\
\hline & & Config. 25 & -4.11 & -21.03 & 0.0000 \\
\hline \multirow[t]{4}{*}{ Config. 19} & 775.59 & Config. 21 & -33.67 & -26.42 & 0.0000 \\
\hline & & Config. 13 & -9.99 & 25.652 & 0.0000 \\
\hline & & Config. 24 & -49.31 & -26.94 & 0.0000 \\
\hline & & Config. 25 & -4.11 & -25.46 & 0.0000 \\
\hline \multirow[t]{3}{*}{ Config. 21} & -33.67 & Config. 13 & -9.99 & -0.773 & 1.0000 \\
\hline & & Config. 24 & -49.31 & -0.5108 & 1.0000 \\
\hline & & Config. 25 & -4.11 & 0.9652 & 0.9998 \\
\hline \multirow{2}{*}{ Config. 13} & -9.99 & Config. 24 & -49.31 & -1.284 & 0.9946 \\
\hline & & Config. 25 & -4.11 & 0.192 & 1.0000 \\
\hline Config. 24 & -49.31 & Config. 25 & -4.11 & 1.4760 & 0.9797 \\
\hline
\end{tabular}


Table 19 shows the Post Hoc pairwise comparison (Tukey) for $\Delta \mathrm{GV} \%$ at various levels of cylinder relative to the occlusal plane. At the occlusal level $(0 \mathrm{~mm})$, image quality significantly differs from $\Delta \mathrm{GV} \%$ values at all other levels. At $2 \mathrm{~mm}$ from the occlusal level (2mm), image quality significantly differs from $\Delta \mathrm{GV} \%$ values at 10 and $15 \mathrm{~mm}$. At levels $4 \mathrm{~mm}$ apical from the occlusal plane ( $4 \mathrm{~mm}, 10 \mathrm{~mm}, 15 \mathrm{~mm}, 25 \mathrm{~mm})$, image quality does not significantly differ from $\Delta \mathrm{GV} \%$ values at any level apical to $10 \mathrm{~mm}$.

Table 19. Post Hoc pairwise comparison (Tukey) for $\Delta \mathrm{GV} \%$ at various level of cylinder

\begin{tabular}{|c|c|c|c|c|c|}
\hline \multirow{2}{*}{$\begin{array}{l}\text { Pairwise Comparison } \\
\text { Baseline }\end{array}$} & \multirow{2}{*}{ Mean } & \multirow{2}{*}{ Level } & \multirow{2}{*}{ Mean } & \multicolumn{2}{|c|}{ Analysis } \\
\hline & & & & T-value & $P$ \\
\hline \multirow[t]{5}{*}{ Occlusal (0mm) } & 42.07 & 2 & 173.09 & 6.764 & 0.0000 \\
\hline & & 4 & 217.40 & 9.052 & 0.0000 \\
\hline & & 10 & 250.45 & 10.758 & 0.0000 \\
\hline & & 15 & 233.36 & 9.876 & 0.0000 \\
\hline & & 25 & 209.17 & 8.627 & 0.0000 \\
\hline \multirow[t]{4}{*}{$2 \mathrm{~mm}$} & 173.09 & 4 & 217.40 & 2.288 & 0.1990 \\
\hline & & 10 & 250.45 & -3.994 & 0.0009 \\
\hline & & 15 & 233.36 & -3.111 & 0.0229 \\
\hline & & 25 & 209.17 & -1.863 & 0.4253 \\
\hline \multirow[t]{3}{*}{$4 \mathrm{~mm}$} & 217.40 & 10 & 250.45 & -1.706 & 0.5274 \\
\hline & & 15 & 233.36 & -0.824 & 0.9632 \\
\hline & & 25 & 209.17 & 0.425 & 0.9982 \\
\hline \multirow[t]{2}{*}{$10 \mathrm{~mm}$} & 250.45 & 15 & 233.36 & -0.883 & 0.9508 \\
\hline & & 25 & 209.17 & -2.131 & 0.2710 \\
\hline $15 \mathrm{~mm}$ & 233.36 & 25 & 209.17 & -1.249 & 0.8127 \\
\hline
\end{tabular}


Table 20 shows the Post Hoc pairwise comparison for $\Delta \mathrm{GV} \%$ at various arch locations. The $\Delta \mathrm{GV} \%$ values in the anterior portion of the arch (Center) are significantly higher from ipsilateral and contralateral locations which are similar.

Table 20. Post Hoc pairwise comparison (Tukey) for $\Delta \mathrm{GV} \%$ at various arch locations

\begin{tabular}{llllll}
\hline $\begin{array}{l}\text { Pairwise } \\
\text { Comparison }\end{array}$ & & & \multicolumn{3}{c}{ Analysis } \\
\hline Baseline & Mean & Level & Mean & T-value & p \\
\hline Center & 245.78 & Ipsilateral & 157.38 & -6.455 & 0.0000 \\
& & Contralateral & 159.62 & -6.291 & 0.0000 \\
Ipsilateral & 157.38 & Contralateral & 159.62 & -0.1636 & 0.9854 \\
\hline
\end{tabular}

Table 21 shows the Post Hoc pairwise comparison for $\Delta \mathrm{GV} \%$ at various resolutions. Overall images performed at a high resolution $(0.076 \mathrm{~mm})$ provide significantly less artifacts than at a low resolution $(0.2 \mathrm{~mm})$.

Table 21. Post Hoc pairwise comparison (Tukey) for $\Delta \mathrm{GV} \%$ at two resolutions.

\section{Pairwise}

Comparison

\begin{tabular}{llllll}
\hline Baseline & Mean & Level & Mean & T-value & p \\
\hline Low & 206.30 & High & 168.88 & 3.346 & 0.0008 \\
\hline
\end{tabular}

Table 22 shows the Post Hoc pairwise comparison for $\Delta \mathrm{GV} \%$ for different arches.

Overall images performed for the maxillary arch have better image quality than for the mandibular arch.

Table 22. Post Hoc pairwise comparison (Tukey) for $\Delta \mathrm{GV} \%$ at two arches

\begin{tabular}{llllll}
\hline $\begin{array}{l}\text { Pairwise } \\
\text { Comparison }\end{array}$ & & & \multicolumn{3}{c}{ Analysis } \\
\hline Baseline & Mean & Level & Mean & T-value & p \\
\hline Maxillary & 170.54 & Mandibular & 204.65 & -3.050 & 0.0023 \\
\hline
\end{tabular}

Table 23 shows the Post Hoc pairwise comparison for $\Delta \mathrm{GV} \%$ according to side of 
HDO in the respective arch. Overall images performed with HDOs bilaterally result in significantly greater reduction in image quality due to scatter $(+\Delta \mathrm{GV} \%)$ than with unilaterally positioned HDOs.

Table 23. Post Hoc pairwise comparison (Tukey) for $\Delta \mathrm{GV} \%$ according to side of HDO

\begin{tabular}{llllll}
\hline $\begin{array}{l}\text { Pairwise } \\
\text { Comparison }\end{array}$ & & & & \multicolumn{3}{c}{ Analysis } & \\
\hline Baseline & Mean & Level & Mean & T-value & p \\
\hline Unilateral & -13.49 & Bilateral & 272.36 & -13.63 & 0.0000 \\
\hline
\end{tabular}

Table 24 shows the Post Hoc pairwise comparison for $\Delta \mathrm{GV} \%$ according to proximity of HDMO to the test cylinder in the arch. Overall images with HDOs adjacent to test cylinder provide significantly worse image quality due to scatter $(+\Delta G V \%)$ than images with HDOs positioned non-adjacent to test cylinder.

Table 24. Post Hoc pairwise comparison (Tukey) for $\Delta \mathrm{GV} \%$ according to proximity of HDMO to test cylinder.

\begin{tabular}{llllll}
\hline $\begin{array}{l}\text { Pairwise } \\
\text { Comparison }\end{array}$ & & & \multicolumn{3}{c}{ Analysis } \\
\hline Baseline & Mean & Level & Mean & T-value & p \\
\hline Non-adjacent & -18.43 & Adjacent & 277.30 & -14.11 & 0.0000 \\
\hline
\end{tabular}


3D Accuitomo 170

Based on the MANOVA from Table $\mathrm{X}$ there is no overall effect from $\mathrm{kV}(90 \mathrm{kV}$ mean $\Delta \mathrm{GV} \%, 128.71 ; 75 \mathrm{kV}$ mean $\Delta \mathrm{GV} \%, 144.77)$ or number of frames (High \# of frames mean $\Delta \mathrm{GV} \%, 139.46$; Low \# of frames mean $\Delta \mathrm{GV} \%, 134.02)$. Therefore data from the these independent variables can be combined and Post Hoc analysis is unnecessary.

Of the total variability, $28.2 \%$ is unexplained (Error SS). The most important factors, in order, are configuration (69\%), proximity (5.9\%), sidedness $(5.3 \%)$, level of cylinder (1.8\%), arch location (0.7\%) and, finally, arch $(0.2 \%)$.

Table 25. 3D Accuitomo 170 Analysis of Variance

\begin{tabular}{ccllll}
\hline Source & $\boldsymbol{D F}$ & \multicolumn{1}{c}{$\boldsymbol{S S}$} & \multicolumn{1}{c}{$\boldsymbol{M S}$} & \multicolumn{1}{c}{$\boldsymbol{F}$} & \multicolumn{1}{c}{ Sig. } \\
\hline Configuration & $\mathbf{1 4}$ & 236794854 & 16913918 & 372.71 & 0.000 \\
Level of Cylinder & $\mathbf{5}$ & 6085359 & 1217072 & 26.82 & 0.000 \\
Arch & $\mathbf{1}$ & 592607 & 592607 & 13.06 & 0.000 \\
Arch location & $\mathbf{2}$ & 2564759 & 1282379 & 28.26 & 0.000 \\
$\mathbf{k V}$ & $\mathbf{1}$ & 139400 & 139400 & 3.07 & 0.080 \\
Frames & $\mathbf{1}$ & 15949 & 15949 & 0.35 & 0.553 \\
Sidedness & $\mathbf{1}$ & 18154387 & 18154387 & 131.79 & 0.000 \\
Proximity & $\mathbf{1}$ & 20152425 & 20152425 & 146.30 & 0.000 \\
Error & $\mathbf{2 1 3 5}$ & 96889199 & 45381 & & \\
Total & $\mathbf{2 1 5 9}$ & $\mathbf{3 4 3 0 8 2 1 2 7}$ & & & \\
\hline
\end{tabular}

DF, degrees of freedom; SS, sum of the squares; $M S$, mean square; F, F value; Sig., statistical significance 


\section{Post Hoc Analysis}

Table 26 shows Post Hoc pairwise comparison for $\Delta \mathrm{GV} \%$ at various $\mathrm{HDO}$ configurations. The analysis shows that overall there are no differences between specific artifacts configurations within 4 groups of increasing $\Delta \mathrm{GV} \%$ :

- Group (Configuration 24, 21, 12, 1, 13, 9, 25, 6, 5, 3 and 2) [Range $\Delta \mathrm{GV} \%,-33.00$ to 9.17],

- Group (Configuration 13, 9, 25, 6, 5, 3, 2, 19 and 18) [Range $\Delta \mathrm{GV} \%,-2.59$ to 81.25], - Configuration $15(\Delta \mathrm{GV} \%, 908.13)$ and configuration $16(\Delta \mathrm{GV} \%, 1041.63)$ are each different from all other configurations.

Identifying trends within this series, taking into consideration the proximity of the test cylinder to the HDO, whether the HDO was uni- or bilateral and number of HDOs indicates that configurations with the worst $\Delta \mathrm{GV} \%$ are those test cylinders adjacent to the HDO or who have the highest number of HDOs overall. 
Table 26. Post Hoc pairwise comparison (Tukey) for $\Delta \mathrm{GV} \%$ for various configurations of Artifacts

\begin{tabular}{|c|c|c|c|c|c|}
\hline \multicolumn{3}{|l|}{ Pairwise } & \multicolumn{3}{|c|}{ Analysis } \\
\hline Baseline & Mean & Level & Mean & T-value & p \\
\hline \multirow[t]{14}{*}{ Config. 1} & -9.92 & Config. 5 & 5.97 & 0.6330 & 1.0000 \\
\hline & & Config. 9 & -1.88 & 0.3201 & 1.0000 \\
\hline & & Config. 2 & 9.17 & 0.7603 & 1.0000 \\
\hline & & Config. 6 & 5.33 & 0.6073 & 1.0000 \\
\hline & & Config. 3 & 8.55 & 0.7358 & 1.0000 \\
\hline & & Config. 12 & -16.97 & -0.2808 & 1.0000 \\
\hline & & Config. 15 & 908.13 & 36.5673 & 0.0000 \\
\hline & & Config. 16 & 1041.63 & 41.8849 & 0.0000 \\
\hline & & Config. 18 & 81.25 & 3.6313 & 0.0225 \\
\hline & & Config. 19 & 79.56 & 3.5640 & 0.0284 \\
\hline & & Config. 21 & -23.36 & -0.5354 & 1.0000 \\
\hline & & Config. 13 & -2.59 & 0.2920 & 1.0000 \\
\hline & & Config. 24 & -33.00 & -0.9194 & 0.9999 \\
\hline & & Config. 25 & -0.75 & 0.3651 & 1.0000 \\
\hline \multirow[t]{13}{*}{ Config. 5} & 14.51 & Config. 9 & -1.88 & -0.3129 & 1.000 \\
\hline & & Config. 2 & 9.17 & -0.127 & 1.0000 \\
\hline & & Config. 6 & 5.33 & -0.0257 & 1.000 \\
\hline & & Config. 3 & 8.55 & -0.1027 & 1.000 \\
\hline & & Config. 12 & -16.97 & 0.9139 & 0.9999 \\
\hline & & Config. 15 & 908.13 & -35.93 & 0.0000 \\
\hline & & Config. 16 & 1041.63 & -41.25 & 0.0000 \\
\hline & & Config. 18 & 81.25 & -2.998 & 0.1539 \\
\hline & & Config. 19 & 79.56 & -2.931 & 0.1820 \\
\hline & & Config. 21 & -23.36 & 1.1684 & 0.9980 \\
\hline & & Config. 13 & -2.59 & 0.341 & 1.0000 \\
\hline & & Config. 24 & -33.00 & 1.552 & 0.9683 \\
\hline & & Config. 25 & -0.75 & 0.26794 & 1.000 \\
\hline \multirow[t]{11}{*}{ Config. 9} & 6.59 & Config. 2 & 9.17 & -0.440 & 1.0000 \\
\hline & & Config. 6 & 5.33 & -0.2872 & 1.000 \\
\hline & & Config. 3 & 8.55 & -0.4157 & 1.0000 \\
\hline & & Config. 12 & -16.97 & 0.6009 & 1.0000 \\
\hline & & Config. 15 & 908.13 & -36.25 & 0.0000 \\
\hline & & Config. 16 & 1041.63 & -41.56 & 0.0000 \\
\hline & & Config. 18 & 81.25 & -3.311 & 0.0641 \\
\hline & & Config. 19 & 79.56 & -3.244 & 0.0784 \\
\hline & & Config. 21 & -23.36 & 0.8555 & 0.9999 \\
\hline & & Config. 13 & -2.59 & 0.028 & 1.0000 \\
\hline & & Config. 24 & -33.00 & 1.240 & 0.9963 \\
\hline
\end{tabular}


Table 26(Continued). Post Hoc pairwise comparison (Tukey) for $\Delta \mathrm{GV} \%$ for various configurations of Artifacts

\begin{tabular}{|c|c|c|c|c|c|}
\hline Pairwise & & & & Analysis & \\
\hline Baseline & Mean & Level & Mean & T-value & p \\
\hline & & Config. 25 & -0.75 & -0.04498 & 1.000 \\
\hline Config. 2 & 11.48 & Config. 6 & 5.33 & -0.153 & 1.0000 \\
\hline & & Config. 3 & 8.55 & -0.025 & 1.0000 \\
\hline & & Config. 12 & -16.97 & 1.0411 & 0.9994 \\
\hline & & Config. 15 & 908.13 & -35.81 & 0.0000 \\
\hline & & Config. 16 & 1041.63 & -41.12 & 0.0000 \\
\hline & & Config. 18 & 81.25 & -2.871 & 0.2099 \\
\hline & & Config. 19 & 79.56 & -2.804 & 0.2445 \\
\hline & & Config. 21 & -23.36 & -1.296 & 0.9941 \\
\hline & & Config. 13 & -2.59 & 0.468 & 1.0000 \\
\hline & & Config. 24 & -33.00 & -1.680 & 0.9395 \\
\hline & & Config. 25 & -0.75 & -0.395 & 1.0000 \\
\hline Config. 6 & 0.27 & Config. 3 & 8.55 & -0.1285 & 1.000 \\
\hline & & Config. 12 & -16.97 & 0.8882 & 0.9999 \\
\hline & & Config. 15 & 908.13 & -35.96 & 0.0000 \\
\hline & & Config. 16 & 1041.63 & -41.28 & 0.0000 \\
\hline & & Config. 18 & 81.25 & -3.024 & 0.1441 \\
\hline & & Config. 19 & 79.56 & -2.957 & 0.1708 \\
\hline & & Config. 21 & -23.36 & 1.1427 & 0.9984 \\
\hline & & Config. 13 & -2.59 & 0.315 & 1.0000 \\
\hline & & Config. 24 & -33.00 & 1.527 & 0.9725 \\
\hline & & Config. 25 & -0.75 & 0.24223 & 1.000 \\
\hline Config. 3 & 7.96 & Config. 12 & -16.97 & 1.0166 & 0.9996 \\
\hline & & Config. 15 & 908.13 & -35.83 & 0.0000 \\
\hline & & Config. 16 & 1041.63 & -41.15 & 0.0000 \\
\hline & & Config. 18 & 81.25 & -2.896 & 0.1982 \\
\hline & & Config. 19 & 79.56 & -2.828 & 0.2315 \\
\hline & & Config. 21 & -23.36 & 1.2712 & 0.9952 \\
\hline & & Config. 13 & -2.59 & 0.444 & 1.0000 \\
\hline & & Config. 24 & -33.00 & 1.655 & 0.9461 \\
\hline & & Config. 25 & -0.75 & 0.37068 & 1.0000 \\
\hline Config. 12 & 29.61 & Config. 15 & 908.13 & 36.8482 & 0.0000 \\
\hline & & Config. 16 & 1041.63 & 42.1658 & 0.0000 \\
\hline & & Config. 18 & 81.25 & 3.9121 & 0.0080 \\
\hline & & Config. 19 & 79.56 & 3.8449 & 0.0103 \\
\hline & & Config. 21 & -23.36 & -0.2546 & 1.0000 \\
\hline & & Config. 13 & -2.59 & 0.5729 & 1.0000 \\
\hline
\end{tabular}


Table 26(Continued). Post Hoc pairwise comparison (Tukey) for $\Delta \mathrm{GV} \%$ for various configurations of Artifacts

\begin{tabular}{|c|c|c|c|c|c|}
\hline \multicolumn{3}{|l|}{$\begin{array}{l}\text { Pairwise } \\
\text { Comparison }\end{array}$} & \multicolumn{3}{|c|}{ Analysis } \\
\hline Baseline & Mean & Level & Mean & T-value & $\mathbf{p}$ \\
\hline \multirow{6}{*}{ Config. 15} & \multirow{6}{*}{19.91} & Config. 24 & -33.00 & -0.6386 & 1.0000 \\
\hline & & Config. 25 & -0.75 & 0.6459 & 1.0000 \\
\hline & & Config. 16 & 1041.63 & 5.32 & 0.0000 \\
\hline & & Config. 18 & 81.25 & -32.94 & 0.0000 \\
\hline & & Config. 19 & 79.56 & -33.00 & 0.0000 \\
\hline & & Config. 21 & -23.36 & -37.10 & 0.0000 \\
\hline \multirow{7}{*}{ Config. 16} & \multirow{7}{*}{18.8} & Config. 13 & -2.59 & 36.275 & 0.0000 \\
\hline & & Config. 24 & -33.00 & -37.49 & 0.0000 \\
\hline & & Config. 25 & -0.75 & -36.20 & 0.0000 \\
\hline & & Config. 18 & 81.25 & -38.25 & 0.0000 \\
\hline & & Config. 19 & 79.56 & -38.32 & 0.0000 \\
\hline & & Config. 21 & -23.36 & -42.42 & 0.0000 \\
\hline & & Config. 13 & -2.59 & 41.593 & 0.0000 \\
\hline \multirow{5}{*}{ Config. 18} & \multirow{5}{*}{17.28} & Config. 24 & -33.00 & -42.80 & 0.0000 \\
\hline & & Config. 25 & -0.75 & -41.52 & 0.0000 \\
\hline & & Config. 19 & 79.56 & -0.067 & 1.0000 \\
\hline & & Config. 21 & -23.36 & -4.167 & 0.0029 \\
\hline & & Config. 13 & -2.59 & 3.339 & 0.0589 \\
\hline \multirow{5}{*}{ Config. 19} & \multirow{5}{*}{18.98} & Config. 24 & -33.00 & -4.551 & 0.0006 \\
\hline & & Config. 25 & -0.75 & -3.266 & 0.0734 \\
\hline & & Config. 21 & -23.36 & -4.099 & 0.0038 \\
\hline & & Config. 13 & -2.59 & 3.272 & 0.0722 \\
\hline & & Config. 24 & -33.00 & -4.483 & 0.0007 \\
\hline \multirow{3}{*}{ Config. 21} & \multirow{3}{*}{15.82} & Config. 25 & -0.75 & -3.199 & 0.0894 \\
\hline & & Config. 13 & -2.59 & -0.827 & 1.0000 \\
\hline & & Config. 24 & -33.00 & -0.3841 & 1.0000 \\
\hline \multirow{3}{*}{ Config. 13} & \multirow{3}{*}{17.83} & Config. 25 & -0.75 & 0.9005 & 0.9999 \\
\hline & & Config. 24 & -33.00 & -1.211 & 0.9970 \\
\hline & & Config. 25 & -0.75 & 0.073 & 1.0000 \\
\hline Config. 24 & 17.32 & Config. 25 & -0.75 & 1.285 & 0.9946 \\
\hline
\end{tabular}

Config., configuration 
Table 27 shows the Post Hoc pairwise comparison (Tukey) for $\Delta \mathrm{GV} \%$ at various levels of cylinder. At the occlusal level $(0 \mathrm{~mm}), \Delta \mathrm{GV} \%$ values are significantly lower than at all other levels. At $2 \mathrm{~mm}$ from the occlusal level $(2 \mathrm{~mm}), \Delta \mathrm{GV} \%$ values are significantly lower than $\Delta \mathrm{GV} \%$ values at $10 \mathrm{~mm}$. At levels $4 \mathrm{~mm}$ and above from the occlusal plane (4mm, 10mm, $15 \mathrm{~mm}, 25 \mathrm{~mm}), \Delta \mathrm{GV} \%$ values are not significantly different from $\Delta \mathrm{GV} \%$ values at any other more distant level.

Table 27. Post Hoc pairwise comparison (Tukey) for $\Delta \mathrm{GV} \%$ at various levels of cylinder

\begin{tabular}{llllll}
\hline $\begin{array}{l}\text { Pairwise } \\
\text { Comparison }\end{array}$ & & & & Analysis & \\
\hline Baseline & Mean & Level & Mean & T-value & p \\
\hline Occlusal (0mm) & 27.65 & 2 & 123.58 & 6.042 & 0.000 \\
& & 4 & 162.24 & 8.477 & 0.000 \\
& & 10 & 192.97 & 10.412 & 0.000 \\
& & 15 & 166.92 & 8.771 & 0.000 \\
$2 \mathrm{~mm}$ & 25 & 147.09 & 7.522 & 0.000 \\
& 123.58 & 4 & 162.24 & 2.435 & 0.1442 \\
& & 10 & 192.97 & 4.370 & 0.0002 \\
& & 15 & 166.92 & 2.729 & 0.0696 \\
$4 \mathrm{~mm}$ & 162.24 & 10 & 147.09 & 1.481 & 0.6766 \\
& & 15 & 192.97 & 1.9351 & 0.3805 \\
$10 \mathrm{~mm}$ & & 25 & 166.92 & 0.2944 & 0.9997 \\
& 192.97 & 15 & 147.09 & -0.9542 & 0.9321 \\
$15 \mathrm{~mm}$ & & 25 & 166.92 & -1.641 & 0.5714 \\
& 166.92 & 25 & 147.09 & -2.889 & 0.0447 \\
& & & 147.09 & -1.249 & 0.8127 \\
\hline
\end{tabular}


Table 28 shows the Post Hoc pairwise comparison for $\Delta \mathrm{GV} \%$ at various arch locations. The $\Delta \mathrm{GV} \%$ at different arch locations are all significantly different from each other with ipsilateral values less than contralateral values which are less than center values overall.

Table 28. Post Hoc pairwise comparison (Tukey) for $\Delta \mathrm{GV} \%$ at various arch locations

\begin{tabular}{llllll}
\hline $\begin{array}{l}\text { Pairwise } \\
\text { Comparison }\end{array}$ & & & & Analysis \\
\hline Baseline & Mean & Level & Mean & T-value & p \\
\hline Center & 182.54 & Ipsilateral & 99.41 & 13.55 & 0.000 \\
& & Contralateral & 128.27 & 7.9 & 0.000 \\
Ipsilateral & 99.41 & Contralateral & 128.27 & 5.65 & 0.0274 \\
\hline
\end{tabular}

Table 29 shows the Post Hoc pairwise comparison between arches for $\Delta \mathrm{GV} \%$. Overall image quality for the maxillary arch is significantly better than the mandibular arch.

Table 29. Post Hoc pairwise comparison (Tukey) for $\Delta \mathrm{GV} \%$ at two arches

\begin{tabular}{llllll}
\hline $\begin{array}{l}\text { Pairwise } \\
\text { Comparison }\end{array}$ & & & & \multicolumn{2}{l}{ Analysis } \\
\hline Baseline & Mean & Level & Mean & T-value & p \\
\hline Maxillary & 120.18 & Mandibular & 153.30 & -3.614 & 0.0003 \\
\hline
\end{tabular}


Table 30 shows the Post Hoc pairwise comparison for $\Delta \mathrm{GV} \%$ according to side of HDO in the arch. Overall images with HDOs bilaterally have significantly poorer quality than those with HDOs unilaterally.

Table 30. Post Hoc pairwise comparison (Tukey) for $\Delta \mathrm{GV} \%$ according to side of HDO in the arch

\begin{tabular}{llllll}
\hline $\begin{array}{l}\text { Pairwise } \\
\text { Comparison }\end{array}$ & & & \multicolumn{3}{c}{ Analysis } \\
\hline Baseline & Mean & Level & Mean & T-value & p \\
\hline Unilateral & 2.870 & Bilateral & 192.661 & -11.48 & 0.0000 \\
\hline
\end{tabular}

Table 31 shows the Post Hoc pairwise comparison for $\Delta \mathrm{GV} \%$ according to proximity of HDO to the test cylinder in the arch. Overall image quality is significantly improved when HDOs are not positioned adjacent to the test cylinder.

Table 31. Post Hoc pairwise comparison (Tukey) for $\Delta \mathrm{GV} \%$ according to proximity of HDO to test cylinder.

\begin{tabular}{llllll}
\hline $\begin{array}{l}\text { Pairwise } \\
\text { Comparison }\end{array}$ & & & & \multicolumn{2}{c}{ Analysis } \\
\hline Baseline & Mean & Level & Mean & T-value & p \\
\hline Non-adjacent & -2.215 & Adjacent & 197.747 & -12.10 & 0.0000 \\
\hline
\end{tabular}




\section{CHAPTER V}

\section{DISCUSSION}

The purpose of this study was to characterize the effect of artifacts due to the presence of coronal high density objects (HDO) in the dental arches on image quality. Since dental amalgam is the most commonly found HDO in the mouth, this project used coronal MOD amalgam preparations to study the beam hardening and scatter effects on radiographic images taken with different exposure parameters.

The model was constructed to simulate the radiographic appearance of the oral cavity as closely as possible. Measured gray values, at specific locations, were compared with gray values of control models (without any HDO) to arrive at $\Delta \mathrm{GV} \%$ which could be statistically compared with each other. $\Delta \mathrm{GV} \%$ was thus used as an index of image quality. $\Delta \mathrm{GV} \%$ value of 0 meant HDOs had no significant effect on the image quality. + $\Delta \mathrm{GV} \%$ values meant that images were lighter than control images and were thus affected by scatter. - $\Delta \mathrm{GV} \%$ values indicated that images were affected by beam hardening and were darker than control images. Using these values as a reference, conclusions were drawn on whether an independent variable affected the image quality.

Each CBCT system (CS 9000 3D, iCAT Next Generation and Accuitomo 170) has differences in data acquisition that make unit comparisons problematic. The CS 9000 has a small, fixed FOV with an incomplete trajectory arc, fixed number of frames but variable $\mathrm{mA}$ and $\mathrm{kV}$; The iCAT Next Generation has a variable FOV, complete trajectory 
arc, variable number of frames but fixed $\mathrm{mA}$ and fixed but high $\mathrm{kV}$. Finally the 3D Accuitomo 170 has a variable FOV, complete trajectory arc, variable number of frames but variable $\mathrm{mA}$ and $\mathrm{kV}$. Overall the CS 9000 3D showed characteristic differences from the iCAT NG and Accuitomo 170, which both demonstrated similar trends.

The CS 9000 3D was the only CBCT unit where most of the variability (78\%) was due to unknown sources. In addition, it was the only unit where the arch (maxilla/ mandible) or sidedness (unilateral/ bilateral) did not have a significant effect on image quality. The worst beam hardening affected areas were occlusal $4 \mathrm{~mm}$ of the ipsilateral cylinder with maximum number of HDOs. Scatter did not play a major role in CS 9000 3D images, except for when HDOs were non-adjacent to the test cylinder and scanned at a high resolution. Contradictory to expectations, a higher $\mathrm{kV}$ resulted in poorer image quality than at a lower $\mathrm{kV}$. Future research could possibly be done to explain this phenomenon.

Images scanned with iCAT NG exhibited greater deterioration of image quality by scatter rather than beam hardening. Occlusal $(0 \mathrm{~mm})-2 \mathrm{~mm}$ of ipsilateral and contralateral test cylinders with adjacent HDOs were most affected by beam hardening while all other levels showed predominantly scatter artifacts. This is probably because all images were scanned at $120 \mathrm{kV}$, which is higher than any other system. Beam hardening effects by HDOs at high $\mathrm{kV}$ is less than at lower $\mathrm{kVs}$. Image quality adjacent to HDOs with bilaterally positioned HDOs scanned at lower resolution were deteriorated by scatter while non- adjacent, unilaterally positioned HDOs scanned at a higher resolution were affected by beam hardening artifacts. Both arches showed significant scatter effects.

For the 3D Accuitomo 170, $\mathrm{kV}$ and number of frames were not found to have a 
significant effect on the quality of images overall. Similar to the iCAT NG, images scanned with this system were more affected by scatter artifacts. Beam hardening artifacts were seen at the occlusal $(0 \mathrm{~mm})-2 \mathrm{~mm}$ of the ipsilateral and contralateral cylinders of adjacent HDOs. Image quality deteriorated by adjacent, bilaterally positioned HDOs due to scatter while image quality due to beam hardening was minimally affected by non- adjacent positioned HDOs. Both dental arches showed significant scatter effects overall.

There are some limitations in this study in that the in-vitro model design does not completely replicate the human dental arch form. In addition this study included only posterior HDOs (dental amalgam) and did not involve other coronal restorative materials such as composite resin, porcelain or complete crowns.

Previous studies that have stated that caries detection is better in CBCT images than intraoral radiographic images have not taken into account the presence of HDOs. ${ }^{(5,9,}$ ${ }^{14,15)}$ This study clearly demonstrates that HDO degrades image quality particularly if they are adjacent to an unrestored coronal area. Clinicians should be aware that this degradation due to the presence of HDO may invalidate the results of these authors. In particular, the results of this study indicate that there is extensive image degradation in the occlusal $4 \mathrm{~mm}$ range of the coronal aspect of the dentition teeth due to beam hardening streak artifacts

There are numerous areas for future research. Additional studies should substitute teeth with various degrees of coronal dental caries for the control cylinders in the current model and investigate the effect of various HDO configurations in dental caries detection. Materials other than dental amalgam should also be used as HDOs. Much of the 
significant variability of the CS 9000 3D is unexplained and not due to CBCT operational parameters or HDO configuration. This was unexpected and should be the subject of further research. In addition unexpected results were found for the 3D Accuitomo 170 in that there was no overall effect from increases in $\mathrm{kV}$ (from $75 \mathrm{kV}$ to $90 \mathrm{kV}$ ) or number of frames, both of which should have improved image quality. Sub-analysis of the data for specific HDO configurations should be performed for this CBCT unit. 


\section{CHAPTER VI}

\section{CONCLUSION}

Within the limitations of this in-vitro study, it can be concluded that:

- HDOs present in the dental arches cause beam hardening and scatter artifacts and reduce image quality due to increasing noise.

- These artifacts are present not only the ipsilateral side but also the contralateral side and anterior region. In other words, HDOs cause artifacts in all areas of the oral cavity, either through beam hardening or scatter artifacts.

- Beam hardening streak artifacts are predominantly seen in the occlusal $4 \mathrm{~mm}$ range of the coronal aspect of the dentition. Scatter artifacts usually predominate more apical to the $4 \mathrm{~mm}$ level.

- Because of the degradation effects of HDO on image quality in the coronal aspect of the dentition, the detection of dental caries on CBCT images in patients with restorations should be viewed with caution. The results of this study suggests that dental caries diagnosis in the presence of HDOs could be either be missed by scatter artifacts or misdiagnosed (false positive) due to beam hardening artifacts. 


\section{REFERENCES}

1. Schulze RKW, Berndt D, D'Hoedt B. On cone-beam computed tomography artifacts induced by titanium implants. Clinical Oral Implants Research. 2010;21(1):1007.

2. Benic GI, Sancho-Puchades M, Jung RE, Deyhle H, Hämmerle CHF. In vitro assessment of artifacts induced by titanium dental implants in cone beam computed tomography. Clinical Oral Implants Research. 2012:n/a-n/a.

3. Naitoh M, Saburi K, Gotoh K, Kurita K, Ariji E. Metal Artifacts From Posterior Mandibular Implants as Seen in CBCT. Implant dentistry. 2013 Apr;22(2):151-4. 4.

Ritter L, Mischkowski RA, Neugebauer J, Dreiseidler T, Scheer M, Keeve E, et al. The influence of body mass index, age, implants, and dental restorations on image quality of cone beam computed tomography. Oral Surgery, Oral Medicine, Oral Pathology, Oral Radiology, and Endodontology. 2009 9//;108(3):e108-e16.

5. Akdeniz BG, Grondahl HG, Magnusson B. Accuracy of proximal caries depth measurements: comparison between limited cone beam computed tomography, storage phosphor and film radiography. Caries research. 2006;40(3):202-7.

6. Tsuchida R, Araki K, Okano T. Evaluation of a limited cone-beam volumetric imaging system: comparison with film radiography in detecting incipient proximal caries. Oral surgery, oral medicine, oral pathology, oral radiology, and endodontics. 2007 Sep;104(3):412-6.

7. Haiter-Neto F, Wenzel A, Gotfredsen E. Diagnostic accuracy of cone beam computed tomography scans compared with intraoral image modalities for detection of caries lesions. Dento maxillo facial radiology. 2008 Jan;37(1):18-22.

8. Tyndall DA, Rathore S. Cone-beam CT diagnostic applications: caries, periodontal bone assessment, and endodontic applications. Dental clinics of North America. 2008 Oct;52(4):825-41, vii.

9. Young SM, Lee JT, Hodges RJ, Chang TL, Elashoff DA, White SC. A comparative study of high-resolution cone beam computed tomography and chargecoupled device sensors for detecting caries. Dento maxillo facial radiology. 2009 Oct;38(7):445-51.

10. Senel B, Kamburoglu K, Ucok O, Yuksel SP, Ozen T, Avsever H. Diagnostic accuracy of different imaging modalities in detection of proximal caries. Dento maxillo facial radiology. 2010 Dec;39(8):501-11.

11. Qu X, Li G, Zhang Z, Ma X. Detection accuracy of in vitro approximal caries by cone beam computed tomography images. Eur J Radiol. 2011 Aug;79(2):e24-7.

12. Kayipmaz S, Sezgin OS, Saricaoglu ST, Can G. An in vitro comparison of diagnostic abilities of conventional radiography, storage phosphor, and cone beam computed tomography to determine occlusal and approximal caries. Eur J Radiol. 2011 Nov;80(2):478-82. 
13. Charuakkra A, Prapayasatok S, Janhom A, Pongsiriwet S, Verochana K, Mahasantipiya P. Diagnostic performance of cone-beam computed tomography on detection of mechanically-created artificial secondary caries. Imaging science in dentistry. 2011 Dec;41(4):143-50.

14. Wenzel A, Hirsch E, Christensen J, Matzen LH, Scaf G, Frydenberg M. Detection of cavitated approximal surfaces using cone beam CT and intraoral receptors. Dento maxillo facial radiology. 2013;42(1):39458105.

15. Sansare K, Singh D, Sontakke S, Karjodkar F, Saxena V, Frydenberg M, et al. Should Cavitation in Proximal Surfaces Be Reported in Cone Beam Computed Tomography Examination? Caries research. 2014 Jan 29;48(3):208-13.

16. Welander U, Nummikoski P, Tronje G, McDavid WD, Legrell PE, Langlais RP.

Standard forms of dentition and mandible for applications in rotational panoramic radiography. Dento maxillo facial radiology. 1989 May;18(2):60-7. 


\section{APPENDIX A}

Configurations show the location (inter and intra arch) of High Density Objects (HDO).

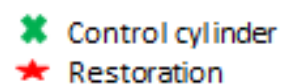

Configuration 1

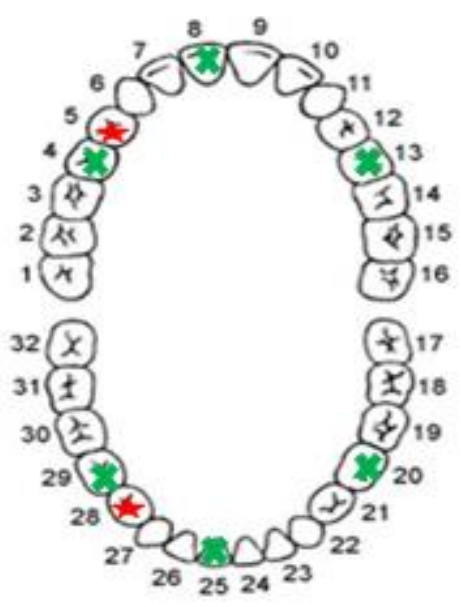

Configuration 3

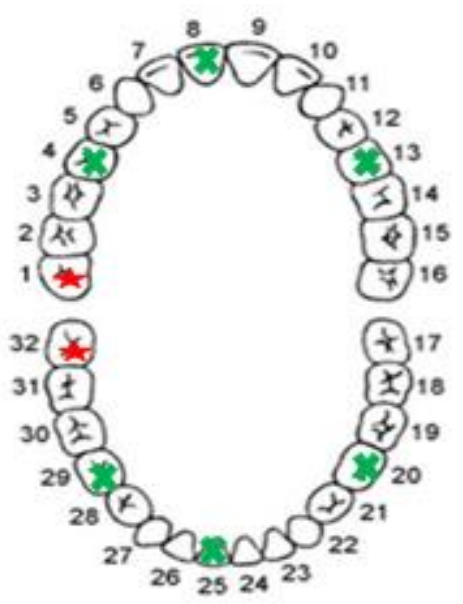

\section{Configuration 2}
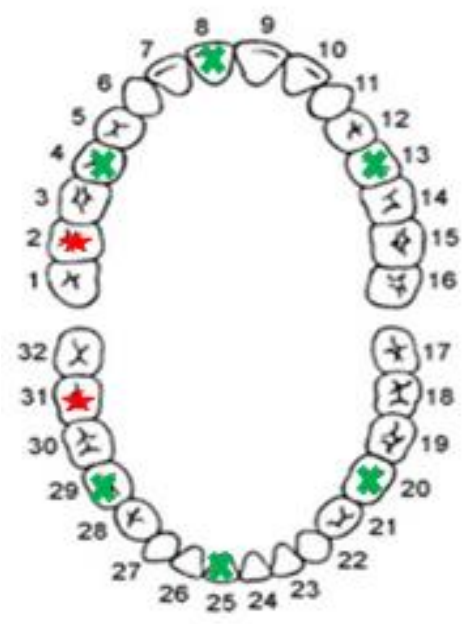

Configuration 5




Configuration 6

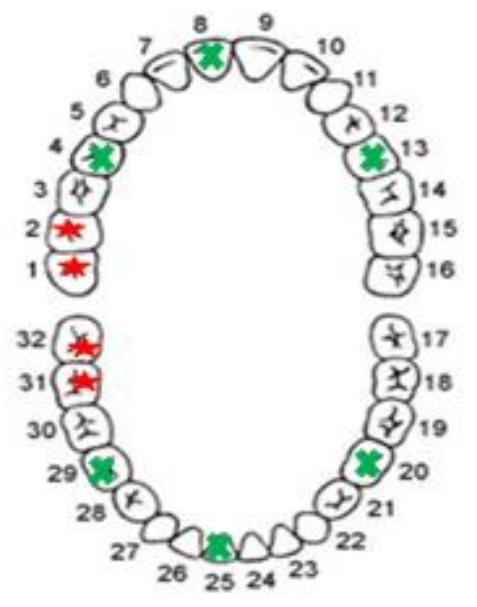

Configuration 12

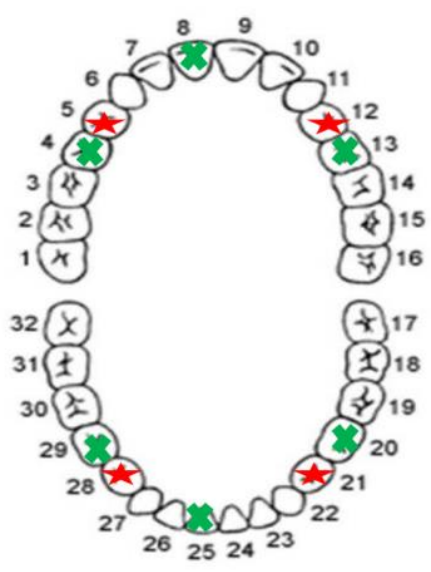

Configuration 9

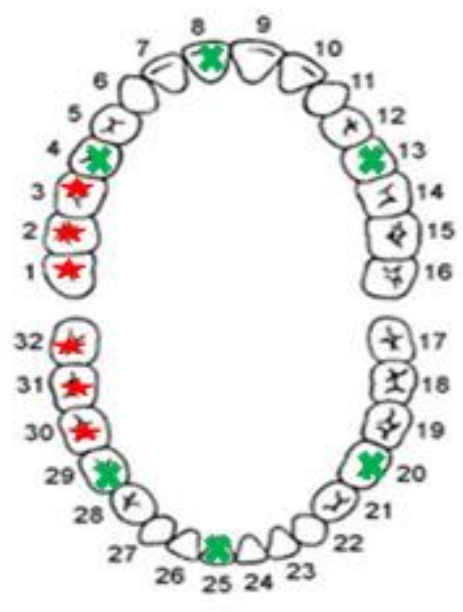

Configuration 13

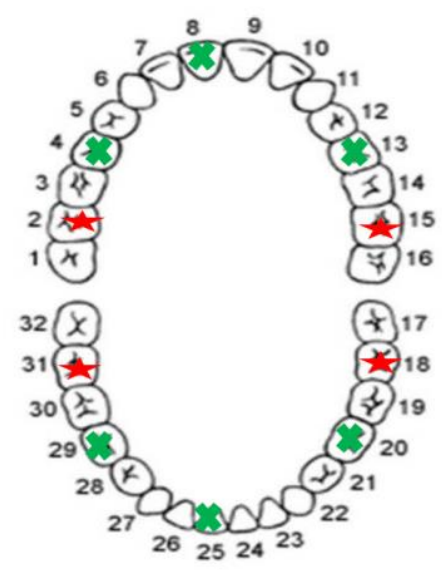


Configuration 15

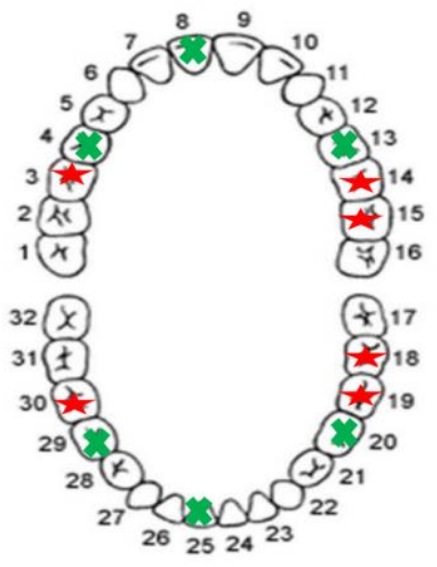

Configuration 18

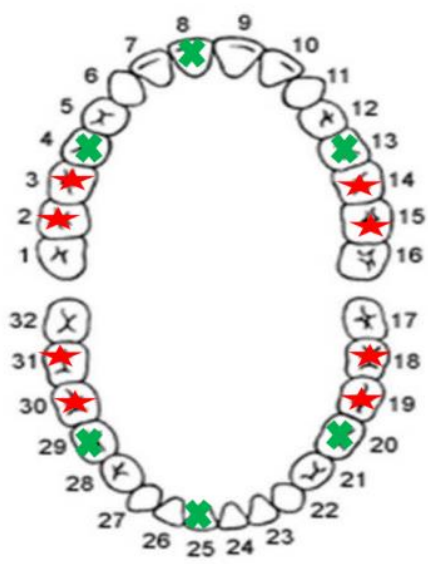

Configuration 16

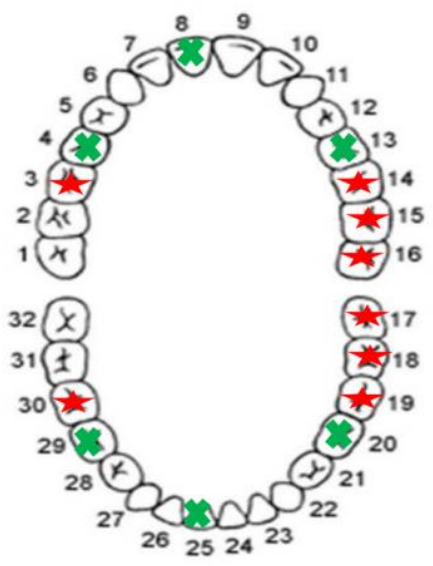

Configuration 19

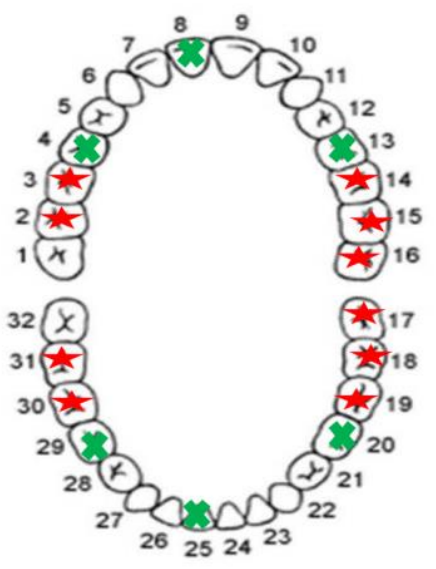


Configuration 21

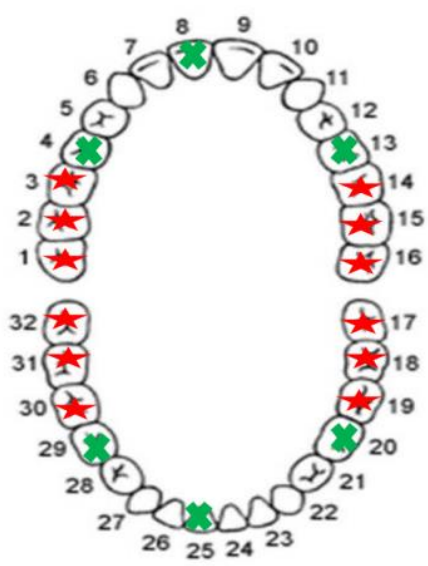

Configuration 25

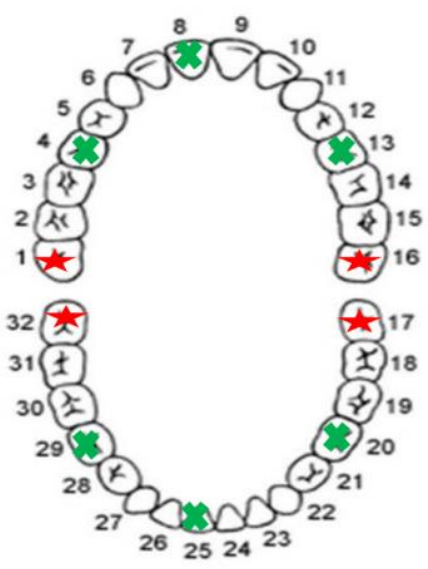

Configuration 24

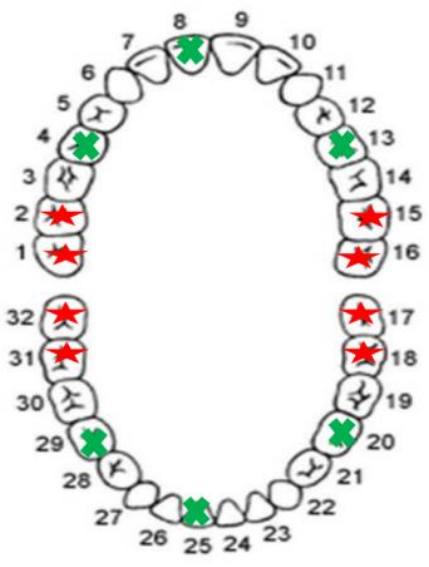




\section{CURRICULUM VITAE}

Name: Mitali Binani

Address: 642 S. 2nd Street, Apt T304,

Louisville,

KY 40202

DOB: December 13, 1987

Education and Training:

- Bachelor of Dental Surgery (B.D.S)

Nair Hospital Dental College

$2006-2011$

- DMD (Batch of 2016)

University of Louisville, School of Dentistry

2014 -till date

Poster and Paper presentation:

- Poster: The Effect of Metallic Restoration Artifacts on Maxillofacial

CBCT images.

2013

Guided by: Farman AG, Scarfe WC, Department of Surgical/Hospital

Dentistry, University of Louisville School of Dentistry

Research!Louisville, 2013

- Paper Presentation: Dawn of Digital Dentistry

2011

Guided by: Dr. Aarti Wadkar, Associate Professor, Department of Prosthodontics, Nair Hospital Dental College

Scientific Rendezvous, Nair Hospital Dental College, 2010-2011 